Alaska Geologic Materials Center Data Report No. 384

\title{
No. 384
}

Boyer, D. 2011, Thin Section Photomicrographs and Descriptions for Mikkelsen Bay St \#13-09-19, W Mikkelsen St \#1, and Sag River St \#1, Lisburne to Total Depth

CD available upon request (183 photos, $148 \mathrm{MB}$ )

Received J anuary, 2011

All data reports may be downloaded free of charge from the DGGS website. 


\section{Description of Mobil Mikkelsen Bay St. \#13-09-19 (50029200550000) Thin Sections (from Alaska State Geological Materials Center):}

\section{$\underline{\text { Echooka Ss. Example Near Pre-Echooka Unconformity }}$}

11,736' Echooka Ss.: well-cemented, ang-subang qtz sand; f-mg with some cg qtzite (Bsmt.) clasts; clay lamins. with organics/tar and pyrite (opaques); well-compacted; tight.

\section{Top of Wahoo/Pre-Echooka Unconformity}

11,752-753' (2 chips): Chip 1: Spic-peloidal chert with occ. euhedral dolo rhombs; contact in T.S. with breccia/conglom.; Chip 2: Breccia/conglom near Pre-Echooka contact (stained); ls. matrix but overall mostly cg-vcg clastics of chert, qtzite. and chalcedony (banded); vf-fg qtz sand within ls. matrix, no porosity.

11,752-53'A (A6228A) a/a but not red stained; vcg-granule-sized clasts of qtz, qtzite and several chert types; one lg. argillite (Bsmt.) clast; tight.

11,752-53'B Spic chert w/ calcite-filled micro-fracs and some qtz silt.

11,753-54' Chert G-MLP with $\lg$ (chert-replaced) bry fronds (covers entire field of view under $5 \mathrm{X}$ lens).

11,754-55' Vcg Bry-echin-occ pelecy dolo lime MRP- 35\% dolo, 5\% chert, 1-2\% qtz sand (silt-vfg); partial grain replacement; some chert in dolo (silicified); poor-fair porosity.

11,766-67' Bry-echin, rare pelecy lime G-MLP- cg-vcg,; chert within echin plate cores; lime mud (no dolo); thin mud coats on many lg. skel. grains; v. tight.

11,780-81' Foram-spic, vf-fg lime G- with bry-echin fines; occ. fg peloids; $10 \%$ angsubang qtz silt-vfg sand; tight.

11,794-95' Bry-echin-foram-pelecy-spic (mixed) G, f-mg; 5\% ang-subang qtz silt-vfg sand; v. well-cmtd, no porosity.

11,969-70' Dolo- vf xln w/ micro-vugs; open HL fracs w/ chert in lg. vug; good porosity.

11,991-92' Lime $\mathrm{W}$ w/ bry-echin-spic skel grains; $2-3 \%$ qtz silt; minor chert replacement within skel grains, esp. echins.; no porosity.

11,998-99' Extremely vf xln dolo- $10 \%$ qtz silt; some calcite-filled HL fracs; some micro-vugs, fair-good porosity.

12,385-86' Vf xln dolo M- sli. silty (qtz); FC's, calcite in micro-vugs but also open vugs; fair-good porosity. 


\section{Mobil Mikkelsen Bay St. \#13-09-19 (cont.)}

12,393-94' Mostly rounded (reworked) echin grains in dolo $M$ matrix (MRP-W) w/ pelecy, rare bry; inter-laced w/ dolo lime mud (any algal?); tar along clay lamins.; no porosity.

\section{Second Set of Unstained Thin Sections:}

11,752-53'B Spic chert G-P with some qtz silt ( 3\%); HL calcite-filled fracs; nonpay.

11,753-54' Chert MRP-W w/ vcg bry-echin-pelecy skel grains (calcite); <1\% qtz; some tar.

11,754-55' Dolo MRP-W- 50\% dolo- f-m xln; 50\% calcite skel grains (bry-echinpelecy): all vcg, esp. bry fronds; some echin plates with chert replacement in cores (centers); poor to fair porosity.

11,766-67' MLP: bry-echin-pelecy, vcg; chert replaces echins. (3\% chert overall); no dolo; tr qtz silt only; no porosity.

11,780-81' Lime G; vfg-fg, spic-forams- \& bry-echin fines, some coated, occ. peloids; $2 \%$ qtz silt-vfg sand; tight.

11,794-95’ Lime G: 3\% qtz silt; $\mathrm{HO} /$ tar along styls.; tight.

11,969-70' Spic dolo W- some lg vugs are chert-filled; vf xln; $1 \%$ qtz; mostly open vugs, good porosity.

11,991-92' Lime $\mathrm{W}$ with cg \& fg bry w/ minor echin \& pelecy skel grains; $2 \%$ qtz silt; no dolo; no porosity.

11,998-99' Dolo W-M- extremely fine $x \ln$ (at 5X magnif.); FO (HL); 2\% qtz silt; common vugs, most open but some chert-filled; good porosity.

12,385-86' Dolo W-M- vf xln; spic; micro-FC; most vugs filled with chert-replaced spics or calcite spar, some filled w/ tar; fair to occ. good porosity.

12,393-94' Dolo MRP-W- mostly rounded, reworked echin. grains; occ. bry \& pelecy grains; $60 \%$ dolo, 40\% calcite (all skel grains); tar-stained clay lamins.; no porosity.

\section{L. Boyer}




\section{Key for Abbreviations:}

$\begin{array}{ll}\text { G } & \text { Grainstone } \\ \text { MLP } & \text { Mud-lean packstone } \\ \text { MRP } & \text { Mud-rich packstone } \\ \text { W } & \text { Wackestone } \\ \text { M } & \text { Mudstone } \\ & \\ \text { Ss. } & \text { Sandstone } \\ \text { Qtz } & \text { Quartz } \\ \text { Qtzite } & \text { Metamorphic quartzite (economic basement) } \\ \text { Gran } & \text { Granule (size of terrig clast) } \\ \text { Pyr } & \text { Pyrite } \\ \text { Dissem } & \text { Disseminated } \\ \text { Cht } & \text { Chert } \\ \text { Chal } & \text { Chalcedony } \\ \text { Bry } & \text { Bryozoan } \\ \text { Echin } & \text { Echinoderm } \\ \text { Pelecy } & \text { Pelecypod (bivalve) } \\ \text { Spic } & \text { Sponge spicules } \\ \text { Fines } & \text { Vfg, almost unrecognizable fragments of common skeletal grains } \\ \text { Donz } & \text { Donezella: a filament algae; look like microscopic "ladders" } \\ \text { Xln } & \text { Crystalline } \\ \text { Microxln } & \text { Microcrystalline } \\ \text { Xstal } & \text { Crystal } \\ \text { Styls } & \text { Stylolites } \\ \text { Anhy } & \text { Anhydrite } \\ \text { Nod } & \text { Nodule } \\ \text { Aliz. Red } & \text { Alizarin red stain for calcite } \\ \text { Lamin } & \text { Laminated or laminations } \\ \text { Terrig } & \text { Terrigenous } \\ \text { Spar } & \text { Calcite spar cement } \\ \text { Frac } & \text { Micro-fracture } \\ \text { FC } & \text { Calcite-filled fracture } \\ \text { FO } & \text { Open or partially open fracture } \\ \text { P \& P } & \text { Porosity \& permeability } \\ \text { Cmt } & \text { Cement } \\ \text { Pel } & \text { Peloid or peloidal } \\ \text { Dissol } & \text { Dissolution } \\ \text { Punky } & \text { V. porous and weakly cemented (can crumble core in your hands!) } \\ \text { T.S. } & \text { Thin section } \\ \text { Orig } & \text { Original } \\ & \end{array}$




\section{Description of ARCO Sag River St. No. 1 (50029200020000) Thin Sections (from Alaska State Geological Materials Center):}

\section{$\underline{\text { Wahoo Zone } 1 \text { (Core Depths: } 9914-10,014 \text { ’ MD) }}$}

9918' Dolo bry-echin-, some pelecy MRP; 60\% dolo w/ abt calcite spar; no visible porosity.

9926' Bry-echin-foram-spic lime G w/ occ. pelecy; some euhedral, lg dolo x-stals; angsubang qtz silt-vfg sand; no porosity.

9927' Bry-echin-,occ. pelecy, v. well-cmtd lime G; tr chert in echin plates, no porosity.

9935' Coated gr. \& ooid vf-fg lime G; grain cores of: bry-echin-pelecy \& occ. peloids; several FC's; no porosity.

9950' Compacted peloid lime $\mathrm{G}$ w/ some veg bry \& echin frags. ( mdst in visual texture); minor chert/clay in fracs. tr qtz silt, rare lg ooids; no porosity.

9954' Oolite with coated grains, m-cg lime G; loosely compacted before early spar cmt; foram centers are common; no porosity.

9965' Coated grain lime G (with occ. true ooids) lime G; f-mg, no porosity.

9976' Coated grain \& ooids lime G; f-mg with/ some cg; no lime mud but not well sorted; minor lg euhedral dolo rhombs, no porosity.

9985, Well-compacted, pelecy-foram-bry-echin-spics (vf-fg) lime G, poss. minor Donezella; also some coated grains with v. thin mud coats; no porosity.

9994' V. sandy lime G to calc qtz ss.; contains calcite spar, bry, echin, vf peloids; 40\% qtz (poss. some clay too); no porosity.

10,005' Coated gr. lime G w/ some true ooids; (grain cores: bry-echin-foram-pelecy); 3$5 \%$ dolo along styls; no porosity.

10,013' Coated grain, f-mg lime $\mathrm{G}$, common cg w/ occ. intraclast; 3-5\% dolo \& qtz silt along styls; FC's; common spar cmt, chert replacement is lacking; no porosity. 


\section{Description of ARCO Sag River St. No. 1 (50029200020000) Thin Sections (from Alaska State Geologial Materials Center):}

\section{Wahoo Zone 7 (Core Depths: 9222-9353' (131' MD)}

9220-30' Oncolite (Osagia-encrusted grains with bryo-echin cores) lime grst; most rims are lime mud with some chert rims and minimal dolimite; common pyrite (finely disseminated), no porosity (2 photos).

9230-40' Silty (quartz), pyritic shale with dehydration cracks; badly plucked thin section (1 photo under crossed-nicols).

9240-50' Oncolite (Osagia) lime grst to MLP (mud-lean pkst) with chert/clay filling in early rim moldic porosity; most rims are lime mud; some bryo-echin are not algal encrusted; minor dolo, common pyrite, no porosity.

9255' (A6302 T.S.) Silty (quartz), compacted dolomite mdst with some coarser X-stals (dolomite overgrowths) and finely disseminated pyrite; no significant vug development; fairly tight.

9255' (TS2) Silty, compacted dolomite, a/a; HL fractures described in core (wellsite notes); stylolite with oil staining and quartz concentration; pyritic with some coarse crystalline dolo overgrowths, fairly tight.

9265' Dolo bryo-echin-pelecy (some coated with dolo rims) MRP (mud-rich pkst); 20$30 \%$ dolo.; some oncolites (partially dolomitized). Dolomitization is incomplete w/o obvious later moldic porosity enhancement; poor porosity.

9280' Dolomitized oncolite grst/pkst (see oncolite fabric "ghosts"); 5\% calcite spar and spar in fracture; both fine and coarse-xlline dolo; well-compacted, appears to be very thin bed based on core descriptions.

9283' Dolo bryo-echin, (some mud coated grains) MLP; 10-20\% dolo (fine \& coarse), some dolo encrusted grains; calcite-filled micro-fracs, poor porosity.

9291' Sli. dolo oncolite \& bryo-echin-pelecy lime MLP; $10-15 \%$ dolo; most encrusted rims are lime mud with limited early moldic porosity filled with mobile chert/clay; poor porosity; $2-3 \%$ internal local (sedimentary?) dip reported in ARCO core description.

9310' Oncolite with some mud-coated bryo-echin-pelecy; $\sim 5 \%$ dolo; most rims are lime mud with some chert/clay filled early moldic porosity; finely dissem pyr; no porosity; very early (pre-compaction) calcite cmt.

9325' Peloid-spic-foram lime grst; tr chert; series of discontinuous calcite-filled microfracs (tension gashes (?)); no porosity. 
9335' Dolo cg bry-echin-pelecy MRP; 40\% dolo, fine \& coarse xlline; no significant chert; no obvious porosity enhancement, poor porosity in T.S.; oil stained in core.

9350' Chert-replaced grst (original fabric probably oncolite-bryo-echin); contact with dolo lime grst near edge of T.S.. Photo taken under crossed-nicols to show texture.

\section{Wahoo Zone 6:}

9360' Spiculitic dolo wkst with peloid "ghosts"; common fine pyrite \& qtz silt; interxlline porosity only, no vugs.

9365' Oncolite (Osagia?) grst; no dolo; all encrusted-grains; some chert filling in early moldic porosity in algal rims; lime mud dominates rims; no porosity.

9378' Peloid (with spar cmt) \& compacted peloid grst with some coated grains; calcitefilled fracs, no porosity. 
Description of ARCO W. Mikkelsen St. No. 1 (50029202780000) Thin Sections (from Alaska State Geological Materials Center):

\section{Comments on Echooka Slides (overlies Wahoo section):}

11,242' Echooka Ss.- tight, common organics w/contorted lamins.

11,252' Echooka Ss.- tight w/ cg-granule-size qtzite. grains.

11,254' Echooka Ss.- a/a, with granule-size qtzite. Clasts (w/ strain lamellae), argillite and spic chert clasts.

11,296' Echooka Ss.- w/ lg shell fragment (pelecy?) and organic lamins.

11,300' Echooka Ss.- silty (qtz) shale.

11,302' Echooka Ss.- tight w/ pyr and tar (opaque).

11,304' Echooka Ss.- a/a, w/ minor calcite spar cmt. and occ. echin. Grains near top of Wahoo ( $1^{\text {st }}$ photo).

\section{Top of Wahoo/Pre-Echooka Unconformity}

11,305' Lime MRP (cg-vcg)- algal-encrusted grains (bry-echin-pelecy cored); chert/clay in rims; no dolo; chert replacement of echin. plate cores; minor qtzite grains (several fused grain clasts); stained.

11,305' Lime MRP- vcg bry, also echin \& pelecy; no dolo; chert in echin plate; FC w/ tar in vugs (partially open); styls. w/ pyr \& tar, no stain.

11,306' Lime MRP (cg-vcg)- bry w/ minor echin \& pelecy; no dolo;; some chert/clyfilled rims; silty; stained.

11,306' Lime W w/ veg bry frond; chert converted to qtz in bry zooecia; $2 \%$ qtz silt; some poss. silicified algal lamins; no stain..

11,307' Peloid lime G- vfg-fg w/ bry-echin-pelecy "fines"; minor chert; 5\% qtz silt; no dolo; stained.

11,307' 95\% Chert nodule w/ spicules; contact with peloidal G, a/a; no stain.

11,310' Peloidal MRP- spic w/ bry-echin-pelecy fines, occ. forams; no dolo; stained.

11,310' Broken chert nodule only; all chert except $\sim 5 \%$ corroded dolo along former styls.; no stain. 


\section{ARCO W. Mikkelsen St. No. 1 (cont.):}

11,312' Chert nodule (60\%) \& mixed peloidal G/MRP with peloidal intraclasts; $1 \mathrm{~g}$ FC; stained but did not hold (spotty).

11,313' Peloidal G w/ bry-echin fines-forams-spic (well-indurated); FC; no stain.

11,315' Peloidal-spic-foram G; no dolo.; minor chert along styls.; no porosity, spotty stain.

11,315' (T.S. 2) Peloidal-spic-foram, poss. Donezella w/ bry-echin-pelecy fines; lg FC; no porosity, unstained.

11,317' Peloidal MRP w/ some bry-echin-pelecy fines \& spics-forams; HL fracs: partially open w/ tar; no porosity, no stain.

11,317' (T.S. 2) Dolo M-W- vf xln, fairly tight w/ few vugs; $5 \%$ qtz silt; chert-clay in small fracs.; poor stain.

11,317' (T.S. 3) Peloidal mixed G inc. spic-forams-bry-echin-pelecy fines (vf-fg); FC's; bad stain job.

11,344' Qtz-chalcedony w/ sharp facets (after anhydrite?) in vf xln dolo matrix (tight but oil-stained); $20 \%$ dolo, $80 \%$ qtz-chal. (A-14689); no stain.

11,350' Dolo W-M- 10\% qtz silt; micro-vugs w/ good interxln porosity; lg FC; no stain.

11,352' Tight dolo $\mathrm{M}$ w/some peloids; $\sim 15 \%$ qtz silt; interxln porosity $\mathrm{w} /$ trace of microvugs only, stained.

11,356' Spic dolo W with radial chert-chalcedony (like Alapah anhy. pseudomorph examples) ; qtzite. clast; $15 \%$ qtz/chal.; some tar in spic vugs; silty; good porosity, no stain.

11,359' Spic vf xln dolo $\mathrm{W}$ w/ elongate vugs; common pyr; $1 \%$ qtz silt; good porosity; no stain.

11,362' V. spic vf xln dolo $\mathrm{W}$; spics filled w/ chert/chalcedony; chert stained maroon (oil or dye?) ; HL FO; interxln porosity, vugs are mostly filled.

11,365' Dolo W- both open \& spar-filled vugs; fine pyr; locally good porosity, no stain.

11,367' Dolo W- spic, a/a w/ chert/qtz in vugs \& open vugs, some with tar; $7 \%$ qtz vfg sand-silt; good porosity; no stain. 


\section{ARCO W. Mikkelsen St. No. 1 (cont.):}

11,370' Dolo W- vugs are open \& calcite-filled; both FO \& FC; poorly stained, good porosity locally.

11,372' Dolo W- with bry-echin-pelecy skel grains; <1\% qtz silt, tight; no red stain.

11,373, Tight spic, argil dolo $\mathrm{W}-\mathrm{w} /$ chal \& calcite-spar in vugs (replacement minerals); interesting chalcedony spicular xstals between 2 styls; no open vugs (poor porosity); 1\% qtz silt, no stain (Alizarin red)).

11,377' Tight, vf $x \ln$ argil dolo $\mathrm{M}$; $\mathrm{v}$ lg chalcedony nodule w/ calcite spar core (similar to Alapah Zone $\mathrm{C}$ descrips.: possible anhydrite pseudomorph?); laminae containing pyr \& organic matter bend around chal. nod.; $2 \%$ qtz silt; not stained.

11,380' Dolo M-occ. W- vf xln, argil w/ tar \& pyr in lamins., a/a; 1-2\% qtz silt; minor spic vugs filled w/ chert-qtz; poor porosity, no stain.

11,382' Dolo M-occ. W- vf xln, argil w/ open styls; 2\% qtz silt; oil-stained but tight; no Aliz. red stain.

11,384' Bry-echin-pelecy, cg G; common styls; also lg forams \& mud/peloid intraclasts; FC's (contorted from later compaction); tr chert in echin plates; no red stain.

11,384' (T.S. 2) Bry-echin-pelecy G- ; well-compacted \& cmtd (tight); styls w/ tar \& pyr; vcg detrital vcg chert clast; no red stain.

11,385' Bry-echin G to MLP- cg; some chalcedony; 5\% chert (within grains); some lg peloids, intraclasts \& pelecys.; no dolo; $2 \%$ ang-subang qtz (f-mg); no porosity; no red stain.

11,385' (T.S. 2) Bry-echin G, a/a- mg-vcg, more in mg-cg; some peloids (lg \& small); $5 \%$ ang qtz (detrital) \& 5\% chert; tight, red stained but did not take well.

11,386' Dolo M- argil, silty, vf xln; 5\% qtz silt; chert replaced spics; FC's \& FO's w/ tar infilling; tight; no red stain.

11,386' (T.S. 2) Peloid-spic-echin-bry MRP- some lime mud intraclasts; cse xln corroded dolo (40\%); cg qtz clasts \& fg qtz sand; chert-cly and lime mud; tight; spotty red stain.

11,389' Bry-echin-pelecy G- fg, mostly chertified (70\%); $10 \%$ f-mg qtz silt; red stain is poor quality; no porosity.

11,391' Dolo W- v. spic; dolo xstals are extremely vf xln; spics replaced by chert $(25 \%)$; 2-3\% qtz silt; FC's; tight w/ red stain. 


\section{ARCO W. Mikkelsen St. No. 1 (cont.):}

11,394' Dolo W-M- fine xln; spic, argil, peloidal; chert and spar fill vugs (none open); poor porosity; no red stain.

11,397' Dolo M- extremely fine $x \ln ; 1-2 \%$ qtz silt; terrig. clay lamins \& micro-drapes; $\operatorname{tr}$ qtz silt; poor porosity but oil-stained; no red stain.

11,400' Dolo M- $1 \mathrm{~g}$ chalcedony cluster (radial extinction) with calcite spar in center ( $\sim 30 \%$ chal nods, surrounded by styls.: poss. zone of anhy. collapse?); $5 \%$ qtz silt; HL FC's; poor porosity; no red stain.

11,401' Silty lime W-M- pyritic, $20 \%$ qtz vfg sand-silt; chert-replaced spics; some dolo xstals throughout; some skel grains; argil; tight; no red stain.

11,403 V. Spic (chert-replaced) Lime MRP- 2-3\% qtz silt; one lg dolo rhomb appears to be replaced by chert; no porosity $\&$ no red stain.

11,403' (T.S. 2) V.Spic (chert replaced) MRP-W; 40\% chert (all spic replaced; rest lime mud; no porosity; red stain for calcite.

11,405' Shale- v. pyr (some replaces spics); 15\% qtz silt to mg; nonpay; no red stain.

11,405' (T.S. 2) (A-14711) Shale- w/ 15\% qtz silt; occ. lg dolo rhombs; pyritic \& siliceous.

11,585' Dolo M- extremely vf xln, argil. with lg. FC's; 5\% qtz silt-vfg sand; tight; no red stain.

11,588' Dolo $\mathrm{M}$ w/ wavy lamins.- fine $x \ln \&$ cse $x \ln$ in voids parallel to lamins.; algal (?) or just soft sediment deformation; no porosity; no red stain.

11,590' Dolo M (laminated); $1 \%$ qtz; tight, no red stain on T.S.

11,591' Dolo M, a/a- 1\% qtz silt (oil stained); fairly tight, no red stain.

11,592' Dolo M, a/a- 2\% qtz silt (oil stain); fairly tight, no red stain.

11,595' Dolo M, a/a- some lg, spar-filled vugs; HL open frac; some microvugs; $1 \%$ qtz silt; sli. improved porosity; no red stain on T.S.

11,597' Collapse feature: mostly clastics with shale, tar, chert nods and terrig. clasts, pyrite (turbulent sample!); nonpay; no red stain.

11,599' Dolo W w/ darker lime mud clasts; $2 \%$ qtz silt; some chert-replaced spics; poor porosity, silicified dolo; no red stain. 


\section{ARCO W. Mikkelsen St. No. 1 (cont.):}

11,602' Dolo $M$, a/a; vf xln with $2 \%$ qtz silt; finely dissem. pyr; poor porosity, no red stain.

11,605' Dolo M- freq. shaly lamins. filled w/ HO/tar; vf xln; $1 \%$ qtz silt; common lime mud (often rounded peloids) clasts; poor porosity; no red stain.

11,608' Dolo M- vf xln; 1-2\% qtz silt; occ spics (chert-replaced); micro-fracs filled with chert; poor porosity; no red stain.

11,612' Dolo M- v. silty-sandy layer, occ. argillite grains; common bry fines (15-20\% qtz silt-vfg sand); calcite-filled fracs (intersecting); poor porosity, no red stain.

11,615, Chert- 1-2\% qtz silt in contact with silicified dolo MRP-W (spiculitic); no porosity, no red stain.

11,617' Spic Dolo W- silicified w/ chert replaced spics; 20\% chert (all spics) \& 10\% qtz silt to vfg sand; poor porosity, Aliz. red stain.

11,620' Dolo M to occ. spic dolo W- $10 \%$ qtz silt; poor porosity, no stain.

Note: All the dolo W/M samples in this series are well-compacted w/ rare vuggy porosity, usually limited interxln porosity.

11,625' Lime $M$ - vf xln dolo) with soft sediment deformation preserved in cse. dolomite M matrix; 3-5\% qtz silt; poor porosity; no red stain.

11,627' Dolomite M- peloid (lg) ghosts of fg dol; both fg \& cg dolo; 10\% qtz silt; fairly tight; no red stain.

11,631' Dolo MRP- silicified, chert in fracs; 3\% qtz silt; 30\% chert encasing lg. dolo. $\mathrm{X}$-stals; common spic \& bry inc v. lg. fronds; poor porosity; no red stain.

11,681' Dolo M, occ. dolo W (Core 6: 50' MD gap between cores)- common oil/tarstained clay wisps; silty (silt-fg-mg qtz); no vugs; 10-15\% qtz; common chert-replaced spics; poor porosity; no red stain.

11,684' Dolo M- silty (20\% qtz silt-vfg sand) w/ occ argil. grains; tarry shale wisps along lamins.; poor porosity; no red stain.

11,687' Dolo M- silty, a/a except f-lmg qtz; poor porosity; no red stain.

11,690' Dolo W- extremely fg dolo w/ spic vugs \& good oil stain; $\sim 10 \%$ ang. qtz silt w/ finely dissem pyr; good vuggy/moldic porosity; no red stain. 


\section{ARCO W. Mikkelsen St. No. 1 (cont.):}

11,693' Dolo W- silicified w/ abt detrital qtz \& detrital chert; also chert-replaced spics; $15 \%$ chert spics; $30 \%$ qtz sand (fg-mg); poor porosity; no red stain.

11,695' (no thin section in collection)

11,697' Dolo M- fine xln w/ argil wispy lamins. coated w/ HO/tar; $1 \%$ silt; occ vugs; some open HL fracs; poor porosity; no red stain.

11,699' Dolo W- fine $x \ln \mathrm{w} /$ common vugs; $1-2 \%$ qtz; oil-stained; good vuggy/moldic porosity; no red stain.

11,701' Dolo W- fine xln; compacted but oil-stained w/ abt spic vugs/molds; good matrix P \& P; spics are both open molds and chert-replaced; tr qtz silt-sand only; no red stain.

11,704' (Bad T.S. with many air bubbles.) Dolo M-W, a/a but with fewer vugs; tr qtz only; fair porosity, good where vuggy; no red stain.

11,707' Dolo M-W, a/a- common vugs in patches=better P \& P; tr qtz only.

11,708' Dolo W- med. xln; abt vugs/molds from spics; occ. spar-filled (largest) vugs; tr qtz only; good P \& P; no red (Alizarin) staining.

11,710' Dolo W- a/a, local patches of micro-vugs; decrease in P \& $\mathrm{P}$ from 11,708' T.S.; no red stain.

11,715, Dolo M-W- a/a, w/ microvugs; some spic molds; tr qtz detrital grains (silt); common finely dissem pyr; fair to good porosity; no red stain.

11,716' Dolo M-W- occ vugs only (rare); FO (partly filled); tr qtz; no red stain.

11,719' Dolo M-W- vf xln w/ tarry styls w/ clay; spics in patches; dolo is silicified (cherty); all spics are chert-replaced; poor porosity; no red stain.

11,722' Dolo M-W- vf-f xln w/ some wispy clay lamins.; finely dissem. pyr; $\sim 1 \%$ qtz; no open vugs, poor porosity; no red stain.

11,725' Dolo M-W- spic; highly burrowed; <1\% qtz; tar stained clay wisps; silicified (chert); poor porosity; no red stain.

11,728' Dolo MRP- bry-echin-no obvious pelecys; 75\% vf xln dolo; $25 \%$ calcite; 1-2\% qtz; spotty red stain; poor porosity.

11,728' Dolo MRP to $\mathrm{W}$ (patches of both)- a/a, in both sples echins are rounded \& abraded from transport; poor porosity; no red stain. 


\section{ARCO W. Mikkelsen St. No. 1 (cont.):}

11,729 (A-14749)' Dolo M- vf xln, argil, pyritic w/ shale wisps; silicified (chert in vugs); spicular pyr (abt); no open vugs; 2-3\% qtz; poor porosity; no red stain.

11,729' Dolo M- a/a, duplicate T.S.; not stained (base of Core 6).

\section{Another set of thin sections from ARCO W. Mikkel. No. 1 (no Alizarin red stain on any ot these):}

11,307' Lime W- with cg-veg bry-echin skel. grains along with bry-echin fines too; not well-sorted (poor); 2-3\% qtz; tight.

11,317' Lime W- v. spic (all chert replaced); FC; series of chert-filled vugs aligned horiz: beach bubbles? EOD significant?; tight.

11,350' Vuggy dolo W-M- silty (3\% qtz); great porosity; poss some grain plucking.

11,353' Vuggy dolo W-M- some spar in vugs; good porosity; 5-7\% qtz silt; orig. fabric: peloidal w/ spics.; poss. some grain plucking.

11,357' Vuggy dolo W-M- burrowed-peloidal "ghosts"; 1\% qtz; common vugs; good porosity.

11,360' Dolo W-M- increase in xstal size from above; mini-vugs only; common styls. w/ detrital qtz \& pyr along contact; $2 \%$ qtz overall; some spar in vugs; fair to good porosity due to decrease in larger vugs.

11,364' Chert- orig. peloid-spic G-P (evidence of orig. fabric); 1\% qtz; nonpay, photo under X-Nicols.

11,367' Dolo W w/ abt vugs- minor calcite spar in some vugs; good expl. of dissol. of calcite in micro-fracs.; tr qtz; great porosity.

11,369' Dolo W-M w/ vugs- vf xln; some calcite spar-filled; a/a, 1\% qtz silt; some plucking of grain, hard to assess \% plucked in prep.; great porosity.

11,386' Calcite spar cmt.- probably from frac. or large vug/cavity; minor (late) chertfilled micro-vugs, no porosity; stained with Aliz. red.

11,393 Spic dolo W- FC; abt vugs/molds (often from spics); 1-2\% qtz; one dissol. microfrac; vf xln.; great P \& P.

11,394' Dolo spic W- a/a; 2\% qtz; vf xln; great P \& P. 


\section{ARCO W. Mikkelsen St. No. 1 (cont.):}

11,399' Dolo W-M- some micro FO (from calcite dissol.); other FC followed by later chert in frac fill vugs; $3 \%$ qtz overall (concentrated in styls.); vuggy in some layers; some spic molds; vf xln.; finely dissem pyr; vuggy, good porosity.

11,594' Dolo M- vf xln; open micro-frac/vertical styl. FO; tr qtz silt; ; abt air bubbles in T.S. glue; fair porosity, no vugs.

11,610' Dolo M- no vugs; <1\% qtz; oil-stained; just interxln (fair) porosity.

11,618' Dolo M-W m-c xln; silty; 3-5\% qtz; some vugs, many spar-filled; peloid "ghosts", some burrows; spics replaced by chert; oil-stained; fair porosity (interxln).

11,622' (Poor thin section (T.S.) with v. thin spots); Dolo M-W-vf xln; 1 open frac; spic vugs/molds=both open and chert-filled; $M \&$ W lamins; oil-stained; good P \& P.

11,628' Dolo M- m-c xln; 5\% qtz silt-vfg sand; no vugs; fair porosity, oil-stained.

$\mathbf{1 1 , 6 8 5}$ ' No rock material on glass slide: all ground away during prep.

11,690' Dolo W- vf xln; spic open vugs/molds; 3-5\% qtz silt/sand; good porosity.

11,692' Dolo W- spic, a/a; vf xln; 2-3\% qtz (less qtz than 11,690' sple); vuggy, good porosity.

11,693 Dolo W- silty \& spic; spics are chert-replaced; 3-5\% qtz; mostly open vugs, some chert-filled, v. good porosity.

11,700' Dolo spic W- vf-f xln; spic vugs (some chert -replaced); most are open; great P \& $\mathrm{P}$.

11,704' Dolo W- med xln; spic; abt vugs (good porosity), some filled w/ chert (most are open).

11,706' Dolo W- spic, very vuggy (“punky” texture); med xln; tr qtz only; great P \& P.

11,707' Dolo W- med-cse xln; v. vuggy (great P \& P); tr qtz silt. Note: T.S. is uneven, too thin in middle and too thick on ends.

11,708' Dolo W- most f-m xln, occ cse xln; spic, very vuggy, great P \& P; tr qtz only.

11,712' Dolo W- spic (distorted molds/vugs during compaction); abt vugs, great P \& P; f$\mathrm{m} x \ln$, occ. cse. xln; tr qtz only. 


\section{ARCO W. Mikkelsen St. No. 1 (cont.):}

\section{Key for Abbreviations:}

\begin{tabular}{ll} 
G & Grainstone \\
MLP & Mud-lean packstone \\
MRP & Mud-rich packstone \\
W & Wackestone \\
M & Mudstone \\
& \\
Ss. & Sandstone \\
Qtz & Quartz \\
Qtzite & Metamorphic quartzite (economic basement) \\
Gran & Granule (size of terrig clast) \\
Pyr & Pyrite \\
Dissem & Disseminated \\
Cht & Chert \\
Chal & Chalcedony \\
Bry & Bryozoan \\
Echin & Echinoderm \\
Pelecy & Pelecypod (bivalve) \\
Spic & Sponge spicules \\
Fines & Vfg, almost unrecognizable fragments of common skeletal grains \\
Donz & Donezella: a filament algae; look like microscopic "ladders" \\
Xln & Crystalline \\
Microxln & Microcrystalline \\
Xstal & Crystal \\
Styls & Stylolites \\
Anhy & Anhydrite \\
Nod & Nodule \\
Aliz. Red & Alizarin red stain for calcite \\
Lamin & Laminated or laminations \\
Terrig & Terrigenous \\
Spar & Calcite spar cement \\
Frac & Micro-fracture \\
FC & Calcite-filled fracture \\
FO & Open or partially open fracture \\
P \& P & Porosity \& permeability \\
Cmt & Cement \\
Pel & Peloid or peloidal \\
Dissol & Dissolution \\
Punky & V. porous and weakly cemented (can crumble core in your hands!) \\
T.S. & Thin section \\
Orig & Original \\
& \\
\hline &
\end{tabular}




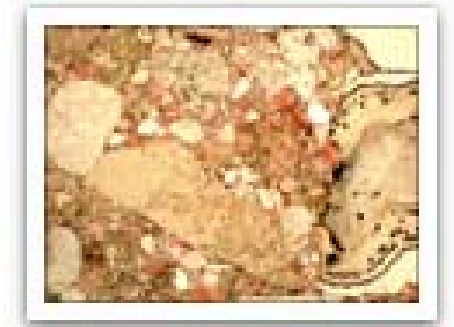

1_Mobil MIKKEL-01 11,752-53...te 2.5X.jpg

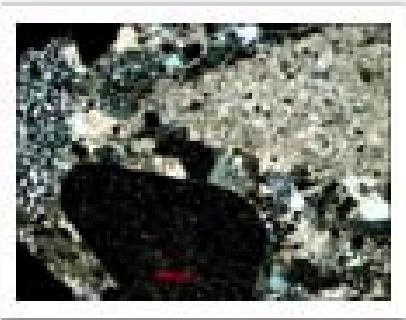

6_Mobil MIKKEL-01 $11,752-53 \ldots$..Is) 2.5X.jpg

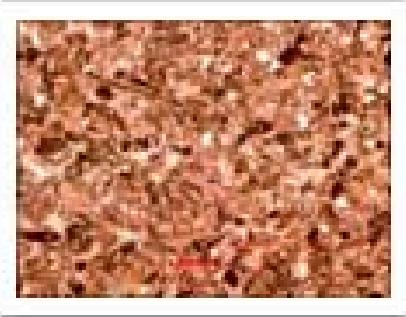

11_Mobil MIKKEL-01 $11,780-81$...fg G 5X.jpg

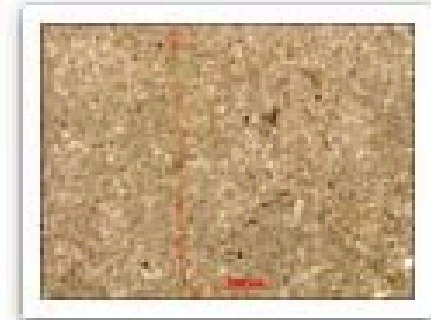

16_Mobil MIKKEL-01 12,385-86...-W 5X.jpg

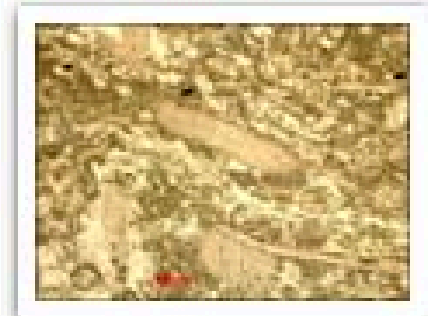

21_Mobil MIKKEL-01 $11,766-67 \ldots \mathrm{GMP} 2.5 X . j \mathrm{pg}$

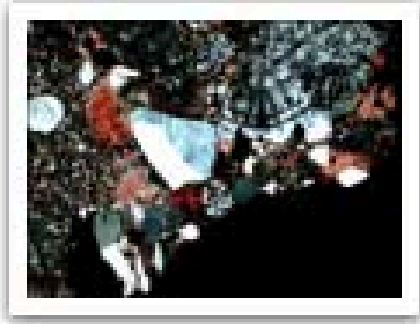

2_Mobil MIKKEL-01 11,752-53...Is) 2.5X.jpg

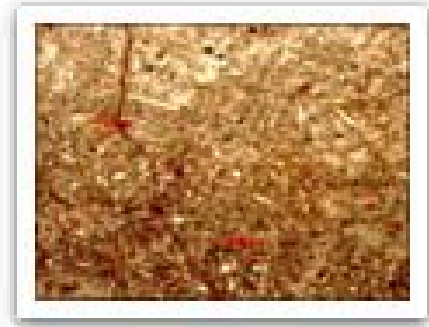

7_Mobil MIKKEL-01 11,752-53...hert 5X.jpg

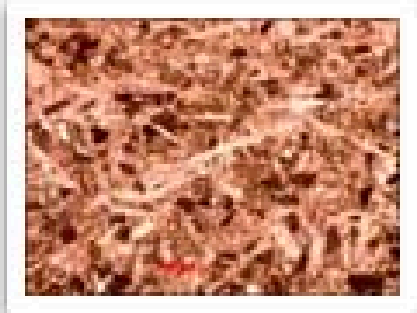

12_Mobil MIKKEL-01 11,794-95...ic G 5X.jpg

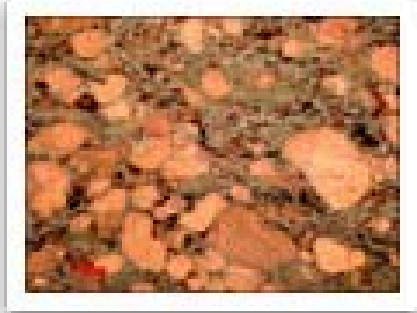

17_Mobil MIKKEL-01 12,393-94_..RP 2.5X.jpg

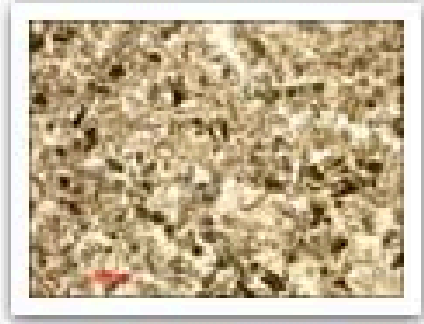

22_Mobil MIKKEL-01 $11,780-81 \ldots$ ic G 5X.jpg

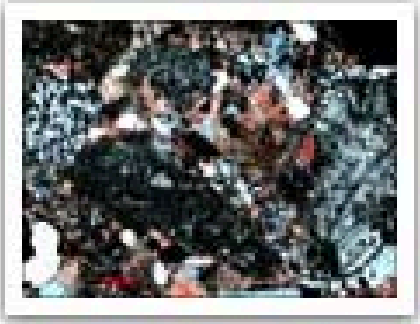

3_Mobil MIKKEL-01 11,752-53...Is) 2.5X.jpg

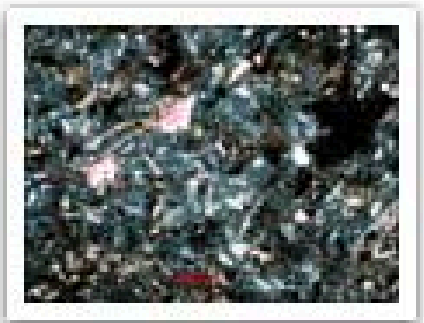

8_Mobil MIKKEL-01 11,753-54...Nicols).jpg

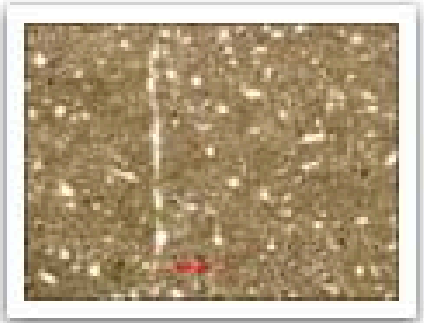

13_Mobil MIKKEL-01 11,969-70...Frac 5X.jpg

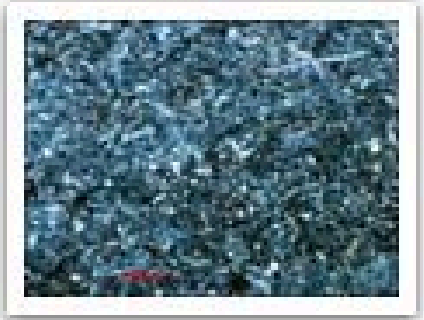

18_Mobil MIKKEL-01 11,752-53...Nicols).jpg

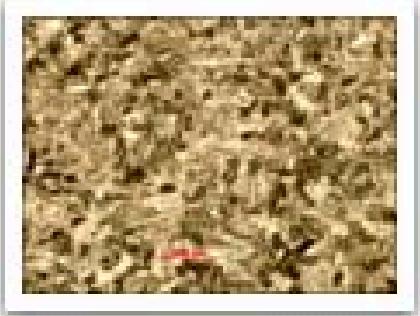

23_Mobil MIKKEL-01 11,794-95 ...ous 5X.jpg

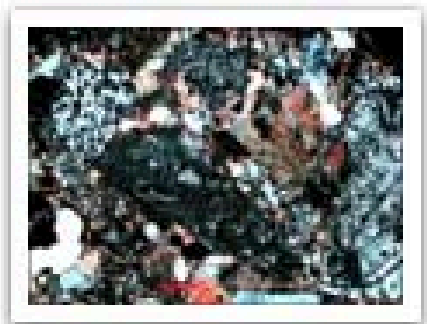

4_Mobil MIKKEL-01 $11,752-53 \ldots$ ols) $5 \mathrm{X} . j p g$

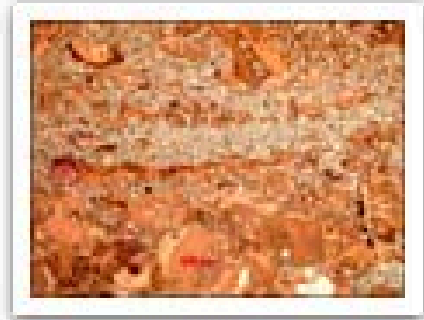

9_Mobil MIKKEL-01 11,754-55 ...ins) 5X.jpg

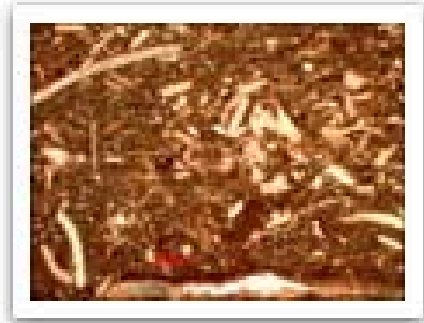

14_Mobil MIKKEL-01 11,991-92 ...in 2.5X.jpg

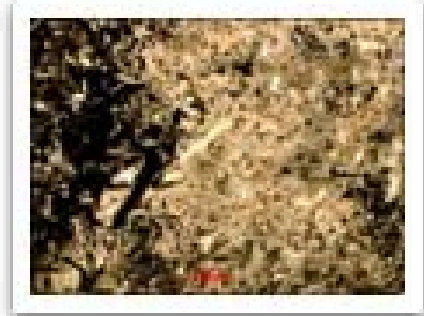

19_Mobil MIKKEL-01 11,753-54...to W 5X.jpg

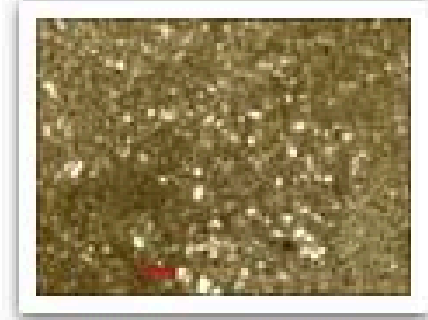

24_Mobil MIKKEL-01 $11,969-70 \ldots$ ggy) $5 X . j p g$

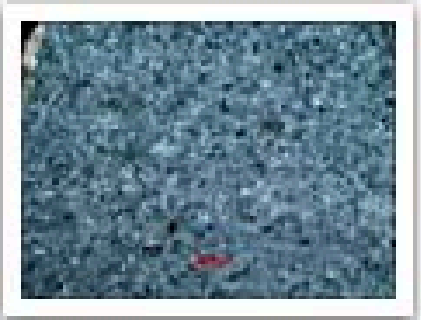

5_Mobil MIKKEL-01 11,752-53...Is) 2.5X.jpg

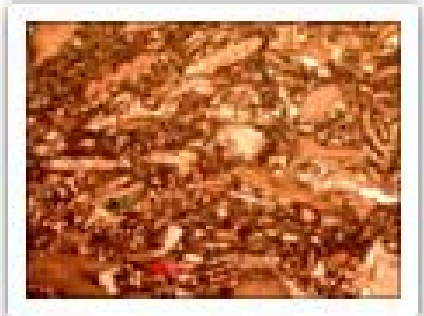

10_Mobil MIKKEL-01 11,766-67...LP 2.5X.jpg

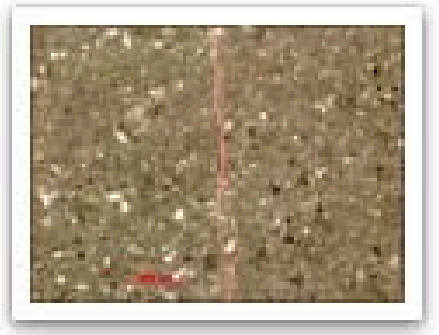

15_Mobil MIKKEL-01 11,998-99...-M 5X.jpg

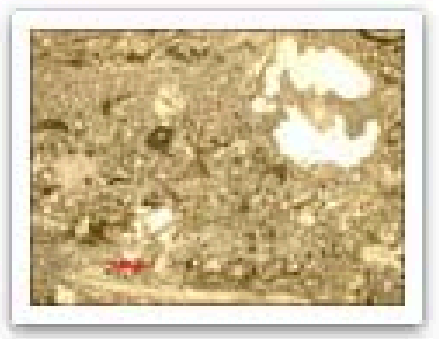

20_Mobil MIKKEL-01 11,754-55....) 2.5X.jpg

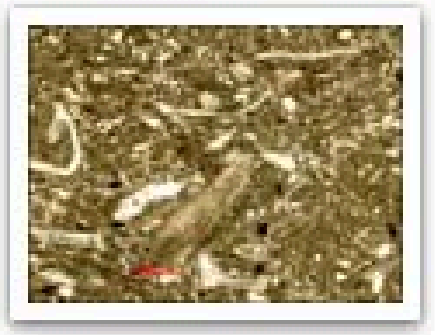

25_Mobil M|KKEL-01 11,991-92 ...ains 5X.jpg 


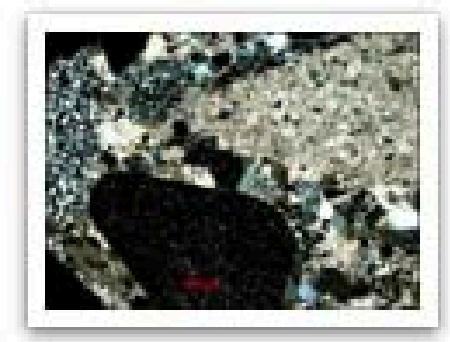

6_Mobil MIKKEL-01

$11,752-53$...Is) $2.5 X . j p g$

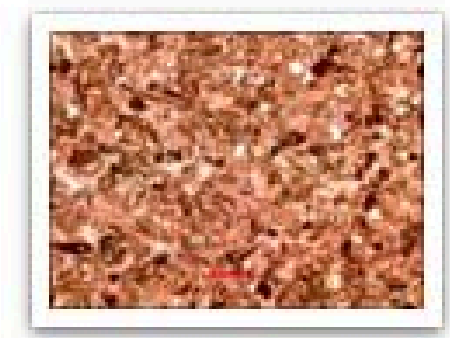

11_Mobil MIKKEL-01 11,780-81...fg G 5X.jpg

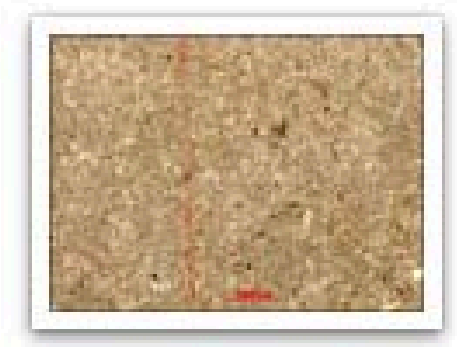

16 Mobil MIKKEL-01 12,385-86...W 5X.jpg

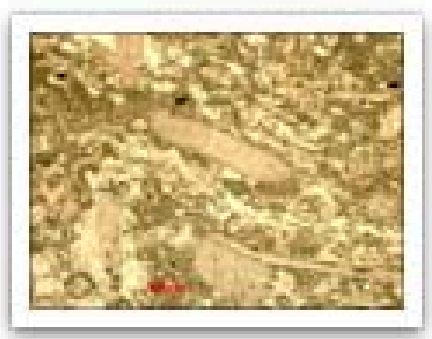

21_Mobil MIKKEL-01 11,766-67...LP 2.5X.jpg

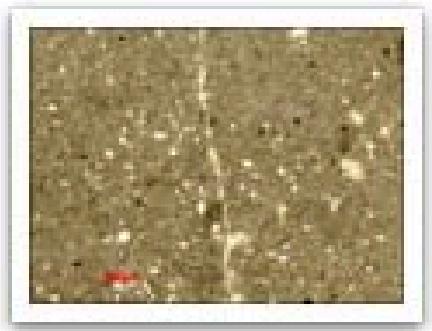

26_MobikMMlKatEReport 384 $11,998-99 \ldots . F O 5 X . j p g$

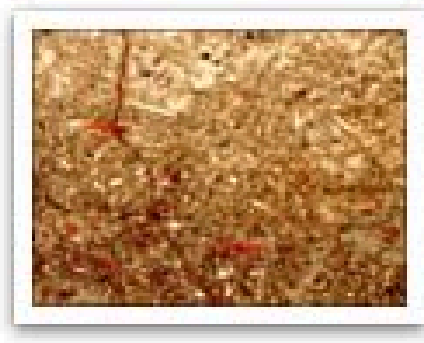

7_Mobil MIKKEL-01 11,752-53...hert 5X.jpg

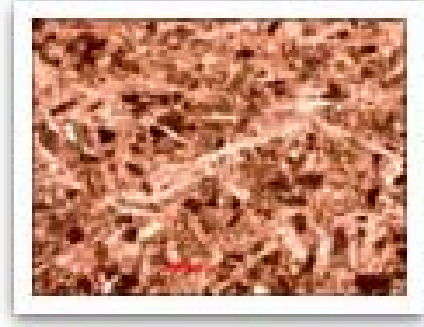

12_Mobil MIKKEL-01 $11,794-95$...ic G 5X.jpg

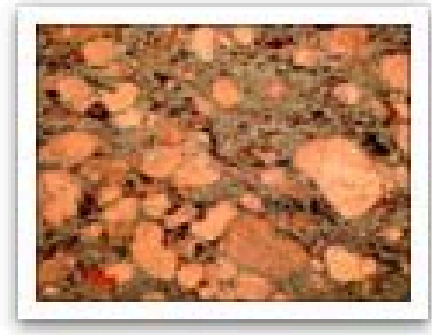

17 Mobil MIKKEL-01 12,393-94 ...RP 2.5X.jpg

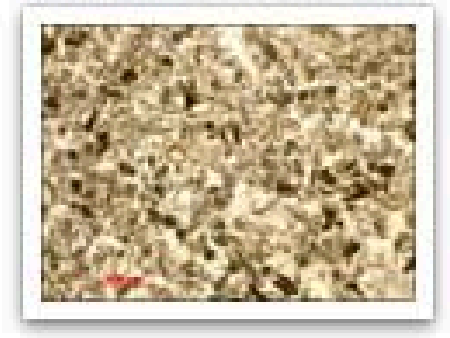

22_Mobil MIKKEL-01 $11,780-81$...ic G $5 X . j p g$

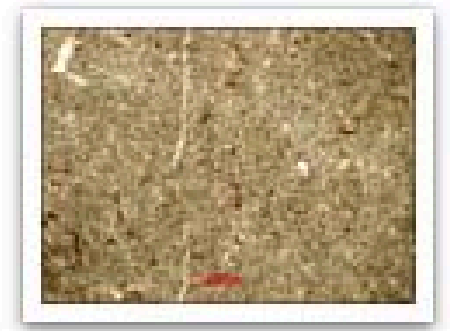

27_Mobil MIKKEL-01 $12,385-86 \ldots-W$ 5X.jpg

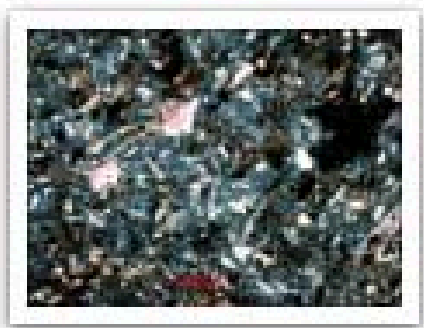

8_Mobil MIKKEL-01 11,753-54...Nicols).jpg

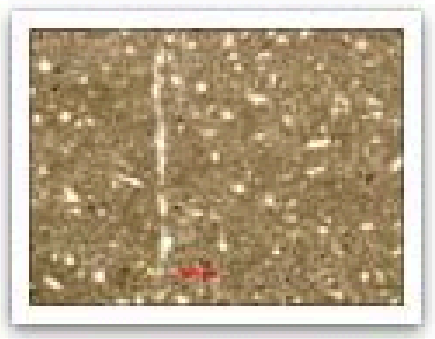

13_Mobil MIKKEL-01 11,969-70...Frac 5X.jpg

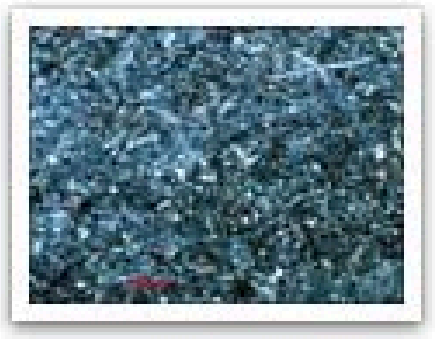

18_Mobil MIKKEL-01 $11,752-53$...Nicols).jpg

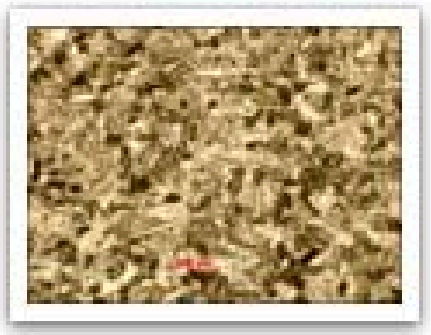

23_Mobil MIKKEL-01 11,794-95...ous 5X.jpg

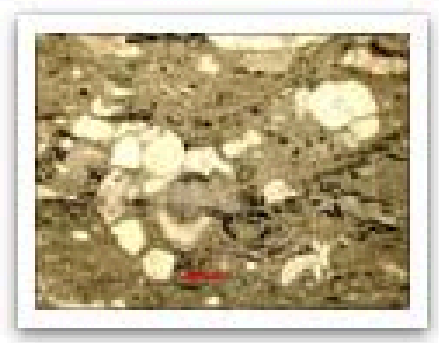

28_Mobil MIKKEL-01 12,393-94...1.) 2.5X.jpg

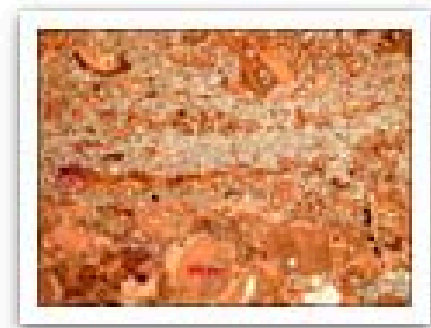

9_Mobil MIKKEL-01 11,754-55 ...ins) 5X.jpg

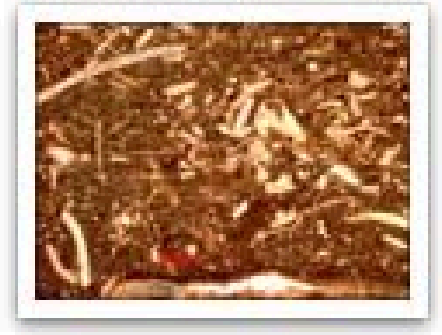

14_Mobil MIKKEL-01 11,991-92...in 2.5X.jpg

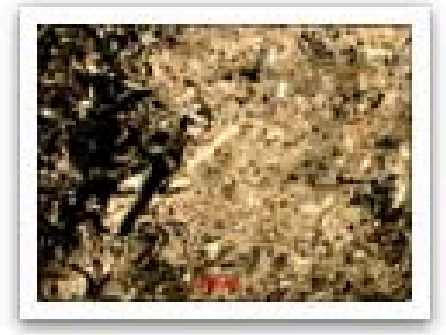

19_Mobil MIKKEL-01 $11,753-54$...to W 5X.jpg

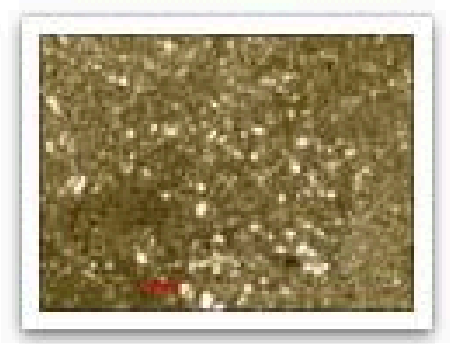

24_Mobil MIKKEL-01 $11,969-70 \ldots g g y) 5 X . j p g$

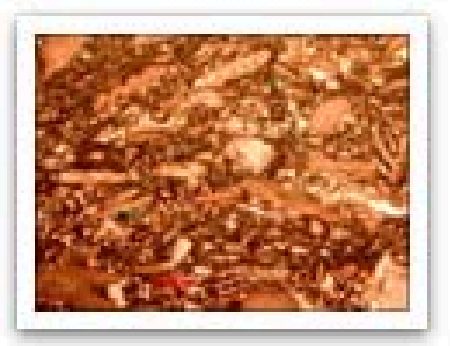

10_Mobil MIKKEL-01 $11,766-67$...LP 2.5X.jpg

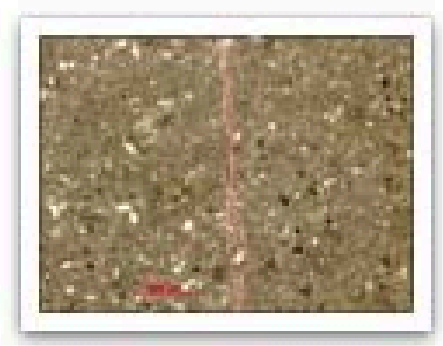

15_Mobil MIKKEL-01 11,998-99...-M 5X.jpg

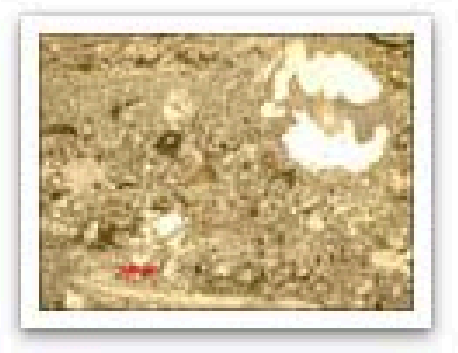

20_Mobil MIKKEL-01 11,754-55...l.) 2.5X.jpg

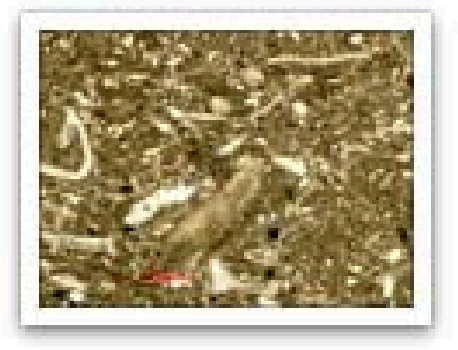

25_Mobil MIKKEL-01 11,991-92...ains 5X.jpg 


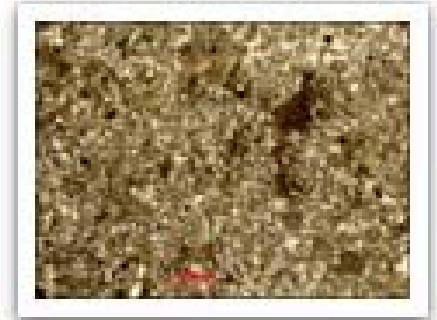

1_SAGR-01 Z1 Core 21 9918' Bry-...) 5X.pg.jpg

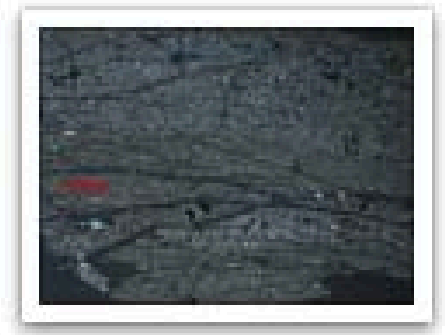

3_SAGR-01 Z7 9230-40'...icols) 5X.jpg

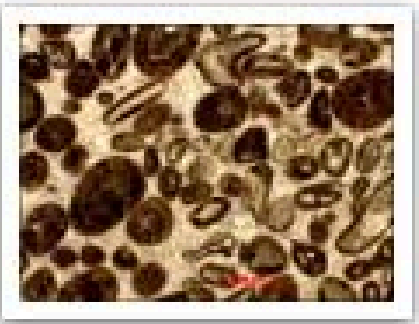

6_SAGR-01 Z1 Core 21 9954' Oolit...e G 5X.jpg

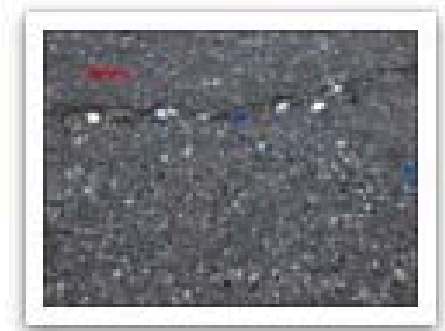

\section{8_SAGR-01 Z7} 9255' (TS2)...ols) 5X.jpg

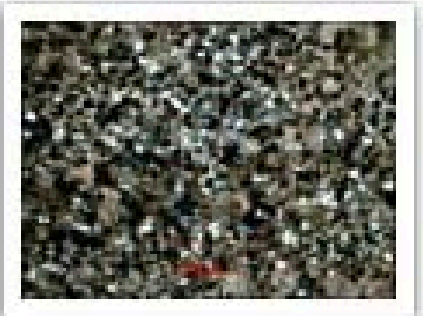

11_SAGR-01 Z1 Core 22

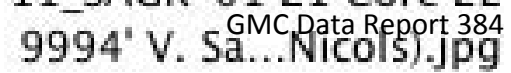

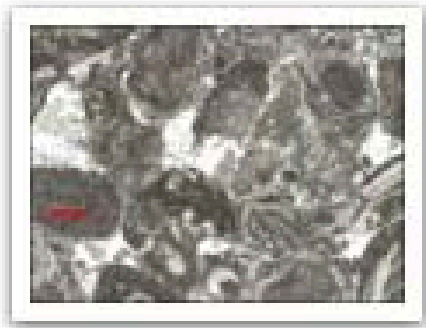

1_SAGR-01 Z7 9220-30'...Rims 5X.jpg

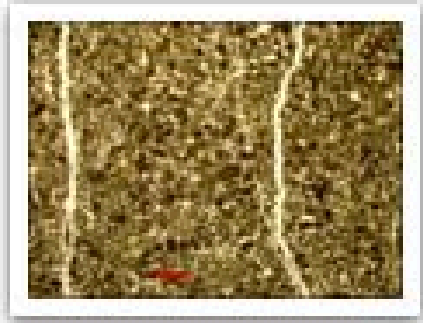

4_SAGR-01 Z1 Core 21 9935'Coat...fg) 2.5X.jpg

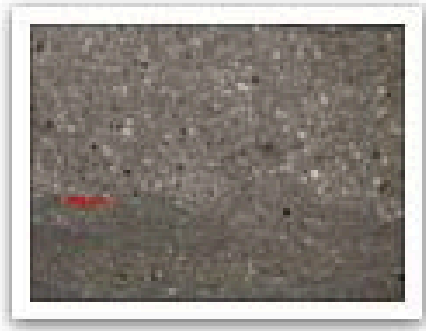

6_SAGR-01 Z7 9255' (A63...dst 5X.jpg

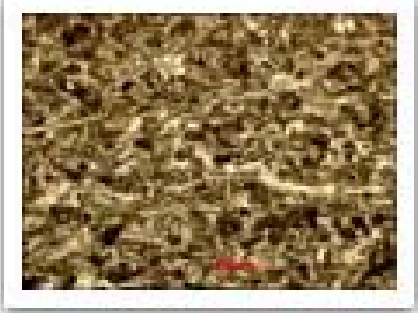

9_SAGR-01 Z1 Core 22 9985' Well...e G 5X.jpg

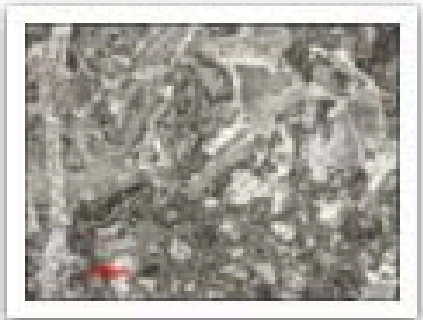

11_SAGR-01 Z7 9283' Sli Dolo Bry...MLP 5X.jpg

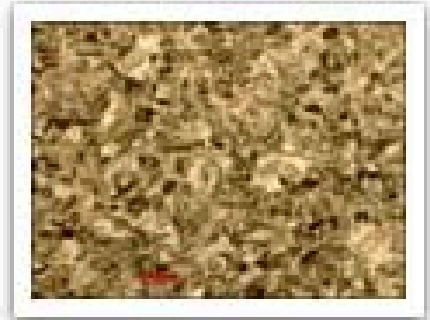

2_SAGR-01 Z1 Core 21 9926' Bry-...(fg) 5X.jpg

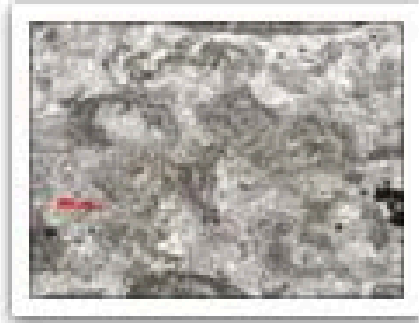

4_SAGR-01 Z7 9240-50'...Rims 5X.jpg

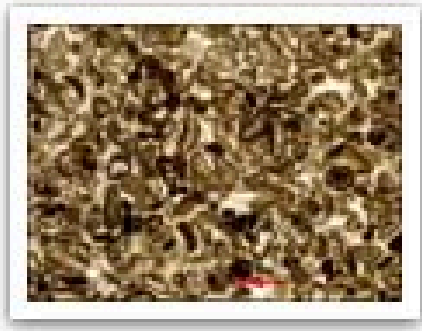

7 SAGR-01 Z1 Core 22 9965' Coat...e G 5X.jpg

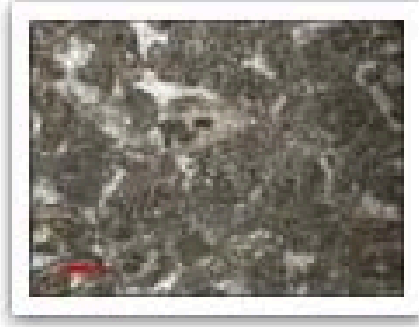

9_SAGR-01 Z7 9265' Dolo Bryo-...MRP 5X.jpg

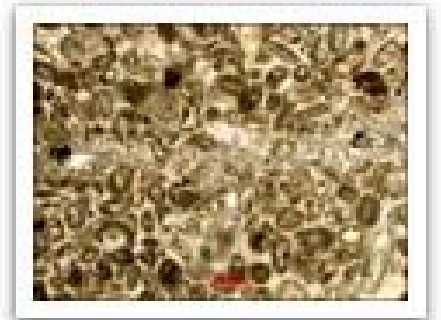

12_SAGR-01 Z1 Core 22 $10,005^{\prime}$ Co...olo) 5X.jpg

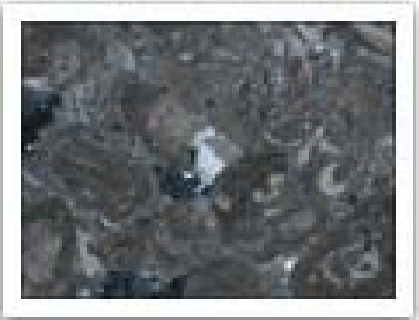

2_SAGR-01 Z7 9220-30'...icols)5X.jpg

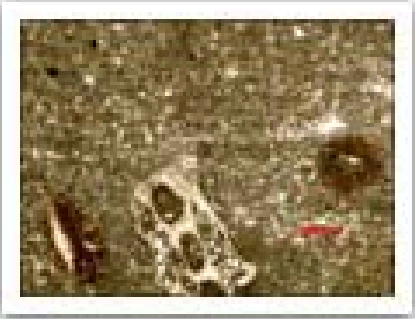

5_SAGR-01 Z1 Core 21 9950' Silty,....rags 5X.jpg

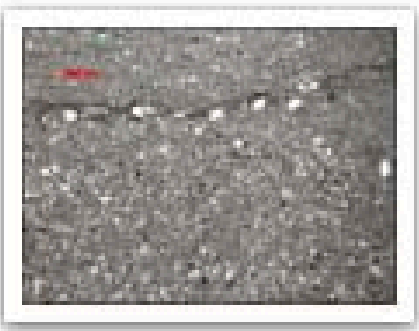

7 SAGR-01 Z7 9255' (TS2 ...Stylo 5X.jpg

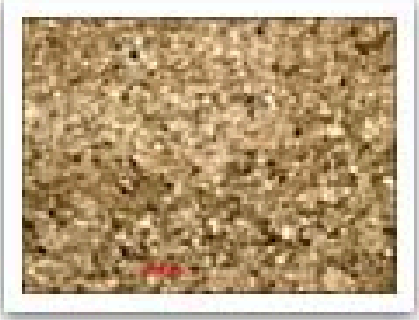

10_SAGR-01 Z1 Core 22 9994' V. Sa...qtz) 5X.jpg

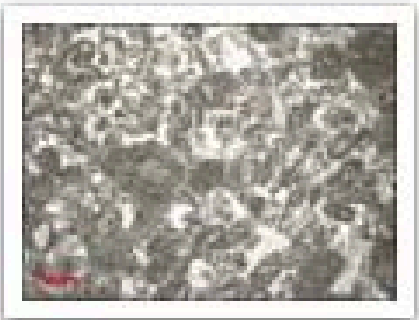

12_SAGR-01 Z7 9291' Sli Dolo En...LP 2.5X.jpg

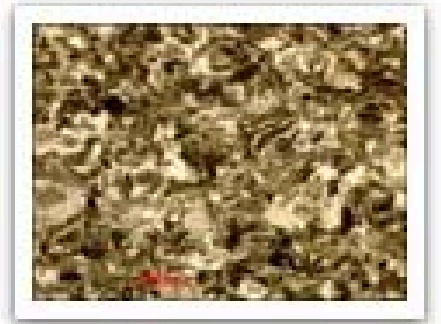

3_SAGR-01 Z1 Core 21 9927' Bry-...(mg) 5X.jpg

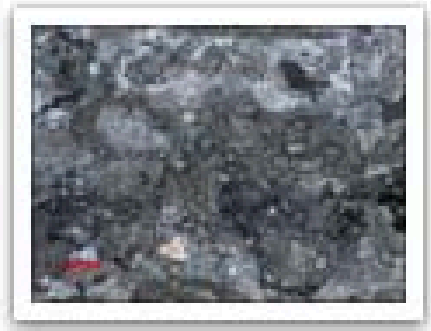

5_SAGR-01 Z7 9240-50'...icols) 5X.jpg

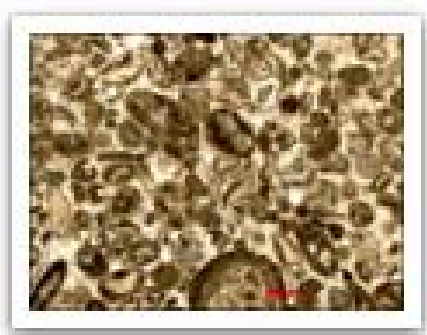

8 SAGR-01 Z1 Core 22 9976' Coat...e G 5X.jpg

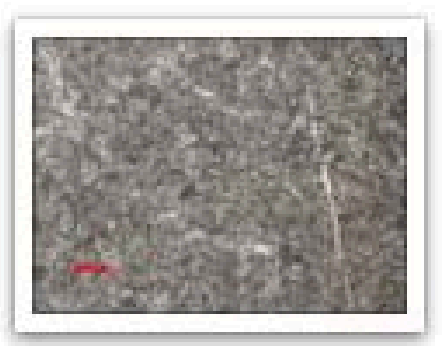

10_SAGR-01 Z7 9280' Fine-Cse X...P-G 5X.jpg

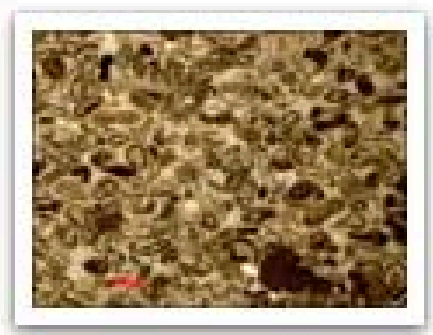

13_SAG-01 Z1 Core 22 $10,013^{\prime}$ Co....010) $5 X . j p g$ 


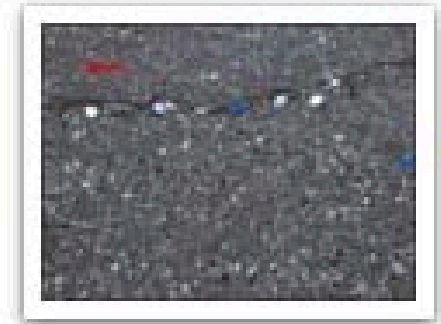

8_SAGR-01 Z7 9255' (TS2)...ols) 5X.jpg

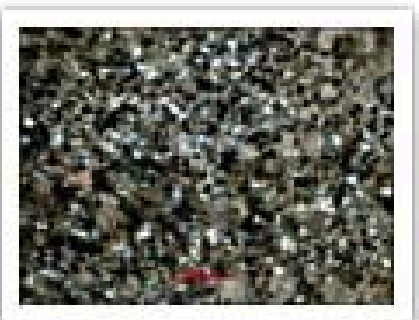

11_SAGR-01 Z1 Core 22 9994' V. Sa...Nicols).jpg

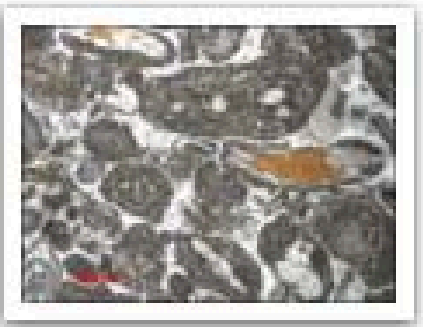

13_SAGR-01 Z7 9310' Sli Dolo En...Grst 5X.jpg

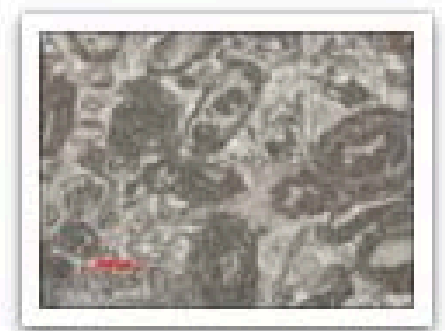

18_SAGR-01 Z6 $9365^{\prime}$ Oncolite (O...Grst 5X.jpg

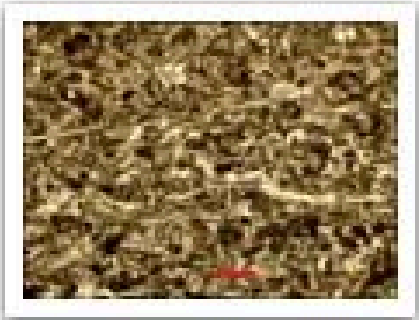

9_SAGR-01 Z1 Core 22 9985' Well...e G 5X.jpg

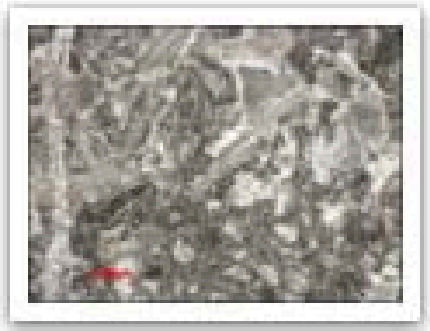

11_SAGR-01 Z7 9283' Sli Dolo Bry...MLP 5X.jpg

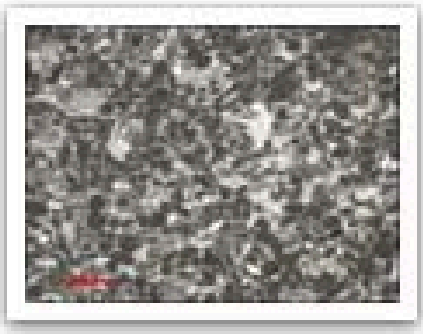

14_SAGR-01 Z7 9325' Peloid-For...Grst 5X.jpg

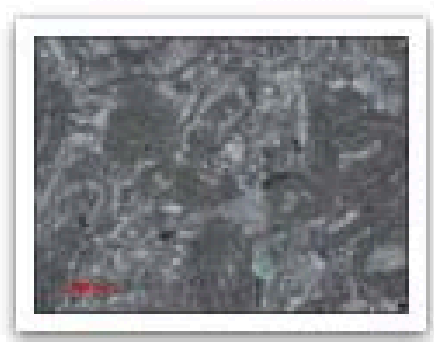

19_SAGR-01Z6 9365' Oncolite (O...ols) 5X.jpg

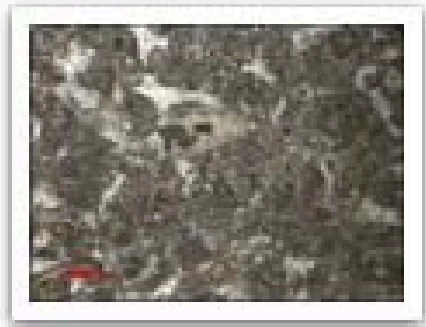

9_SAGR-01 Z7 9265' Dolo Bryo-...MRP 5X.jpg

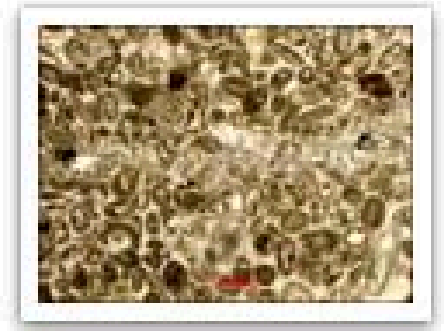

12_SAGR-01 Z1 Core 22 10,005' Co...olo) 5X.jpg

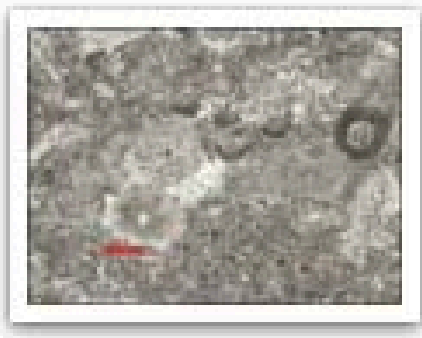

15_SAGR-01 Z7 9335' $40 \%$ Dolo....MRP 5X.jpg

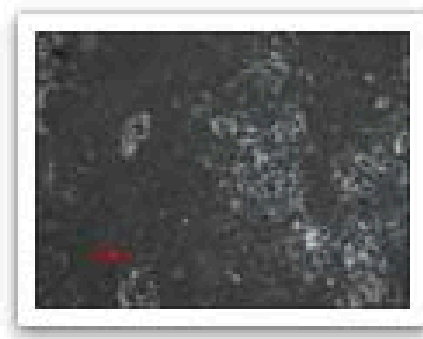

20_SAGR-01 Z6 9378' Peloid \& C...rst 2.5X.jpg
10_SAGR-01 Z1 Core 22 $9994^{\prime}$ V. Sa...qtz) 5X.jpg

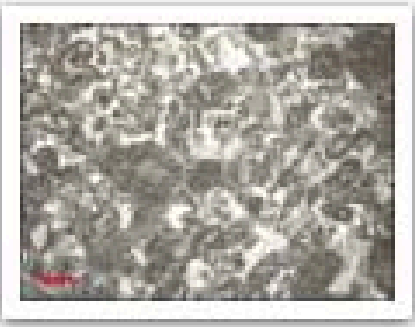

12_SAGR-01 Z7 9291' Sli Dolo En...LP 2.5X.jpg

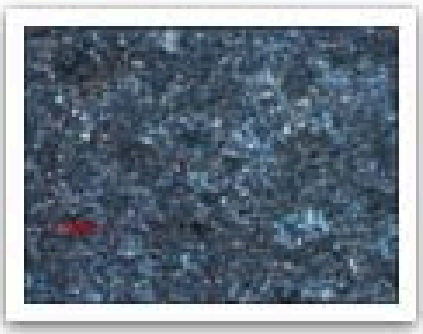

16_SAGR-01 Z7 9350' Chert-Repl...ols) 5X.jpg

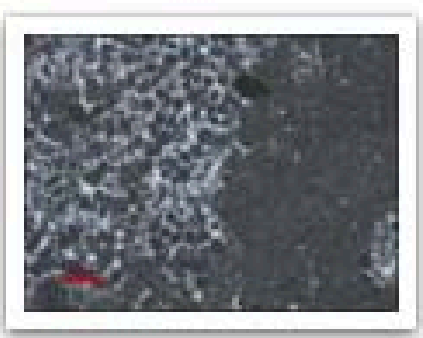

21_SAGR-01 Z6 9378' Peloid, Coa...Grst 5X.jpg
10_SAGR-01 Z7 9280' Fine-Cse X...P-G 5X.jpg

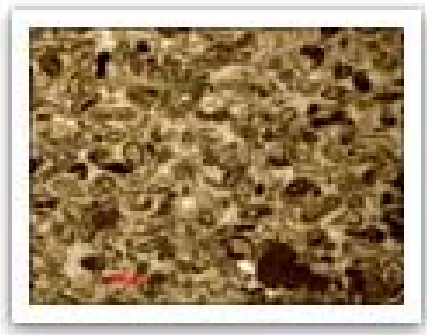

13_SAG-01 Z1 Core 22 $10,013^{\prime}$ Co...olo) 5X.jpg

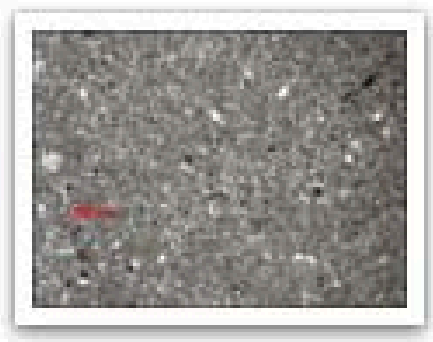

17_SAGR-01 Z6 9360' Spic Dolo...hosts 5X.jpg 


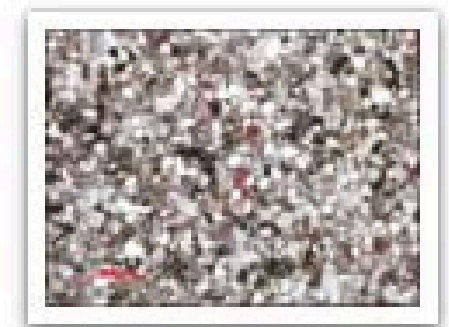

1_WMIKKEL-01 Core 2 11,304' Ec...ntact 5X.jpg

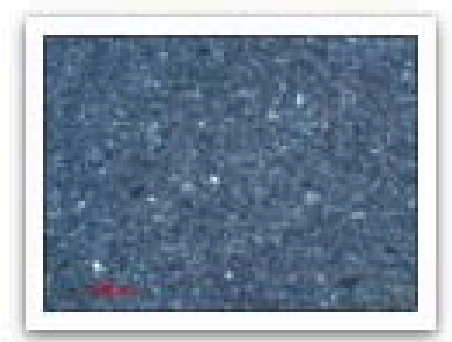

6_WMIKKEL-01 Core 2 $11,307^{\prime}$ Ch...Nicols) .jpg

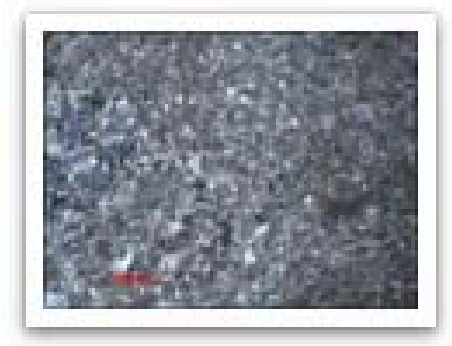

11_WMIKKEL-01 Core 3 11,313' Spi...Nicols).jpg

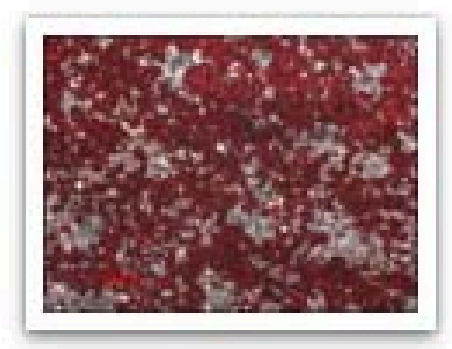

16_WMIKKEL-01 Core 3 $11,317^{\prime}$ Pel...tained) .jpg

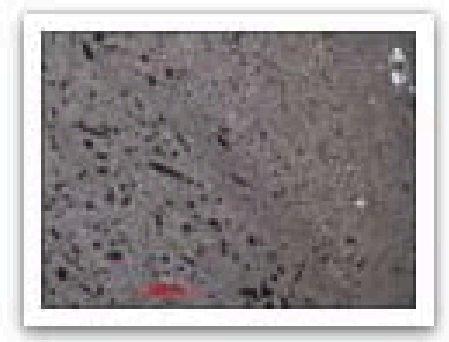

21_WMIKKEL-01 Core 4 11,356' SpGMMPatadepporf j894

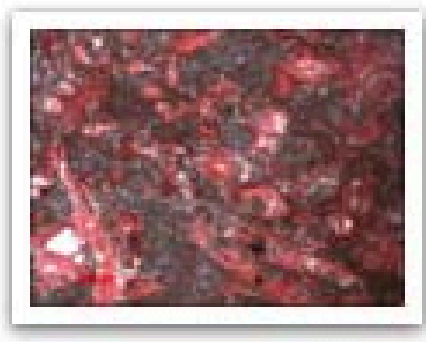

2_WMIKKEL-01 Core 2 11,305' Al...P 5Xjpg.jpg

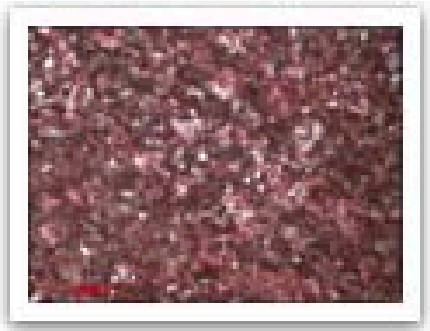

7_WMIKKEL-01 Core 2 11,307' Mi...Grst. 5X.jpg

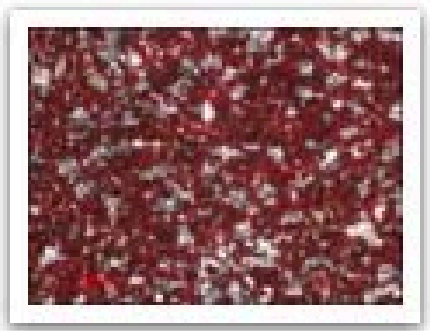

12_WMIKKEL-01 Core 3 11,315' Pel...d stain).jpg

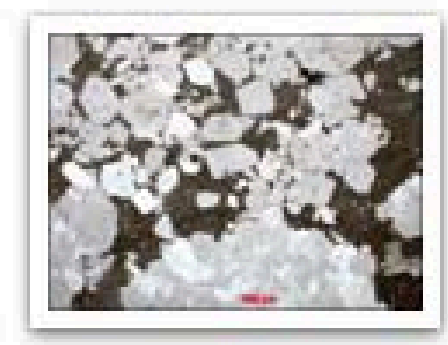

17 WMIKKEL-01 Core 4 $11,344^{\prime}$ Qt...plain It.jpg

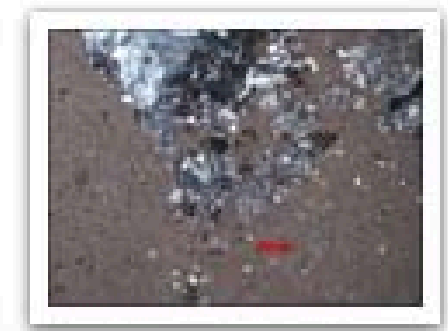

22_WMIKKEL-01 Core 4 11,356' Spi...Nicols).jpg

\section{3_WMIKKEL-01 Core 2} $11,305^{\prime} \mathrm{Br}$...in) 2.5X.jpg

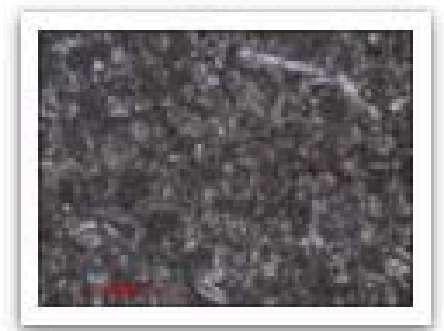

8_WMIKKEL-01 Core 3 11,310 ' Spi...tram In.jpg

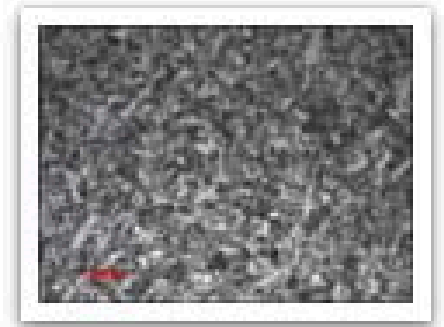

13_WMIKKEL-01 Core 3 11,315' Pel...tram In.jpg

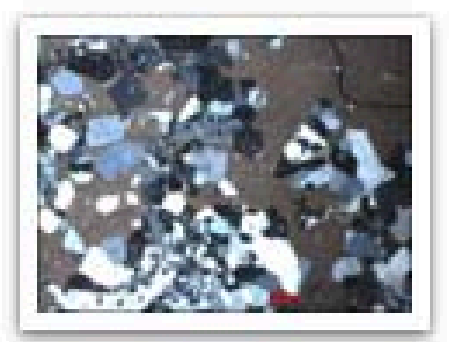

18_WMIKKEL-01 Core 4 $11,344^{\prime}$ An...Nicols) .jpg

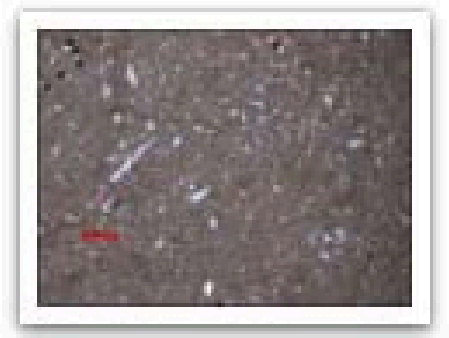

23_WMIKKEL-01 Core 4 $11,359^{\prime}$ Sp...lo W 5X.jpg
4_WMIKKEL-01 Core 2 11,306 ' Sil...Grst. 5X.jpg

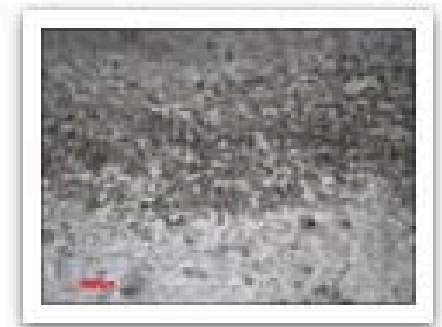

9_WMIKKEL-01 Core 3 $11,310^{\prime}$ Ch...tram In.jpg

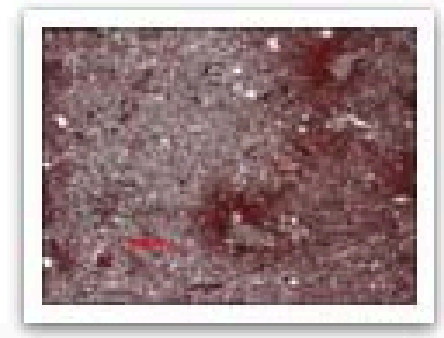

14_WMIKKEL-01 Core 3 11,317' Do...x In 10X.jpg

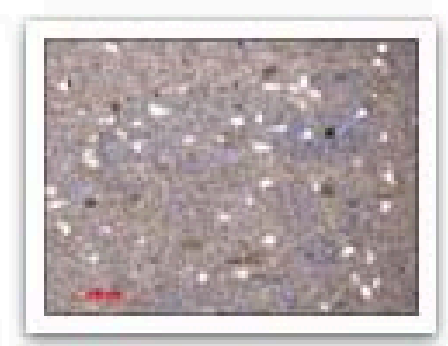

19 WMIKKEL-01 Core 4 11,350 ' Do...pic.) 5X.jpg

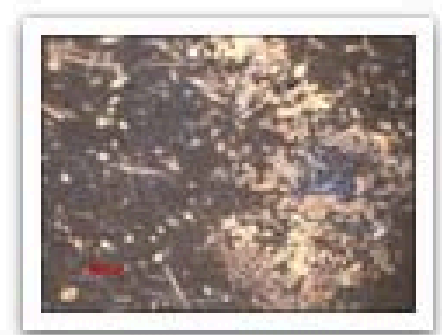

24_WMIKKEL-01 Core 4 11,362 ' Sp...ced) $5 \mathrm{X} . j \mathrm{pg}$
5_WMIKKEL-01 Core 2 11,306' Br...in) 2.5X.jpg

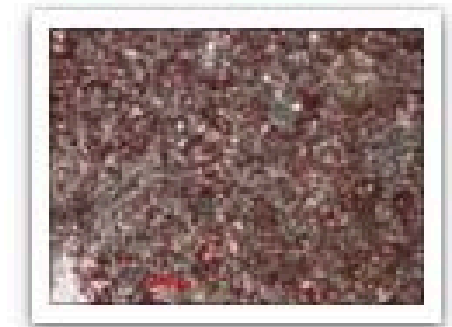

10_WMIKKEL-01 Core 3 11,312' Pel...tact 5X.jpg

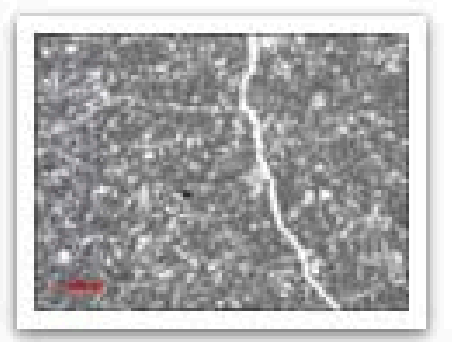

15_WMIKKEL-01 Core 3 11,317' Li...no stain.jpg

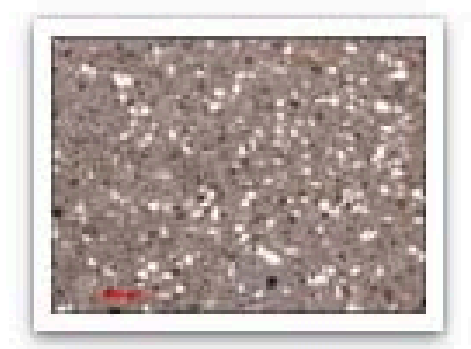

20_WMIKKEL-01 Core 4 $11,352^{\prime}$ 'Silt.... M 5X.jpg

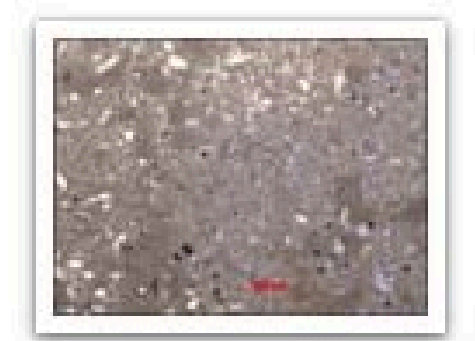

25_WMIKKEL-01 Core 4 $11,365^{\prime}$ Sp11...625s 5X.jpg 


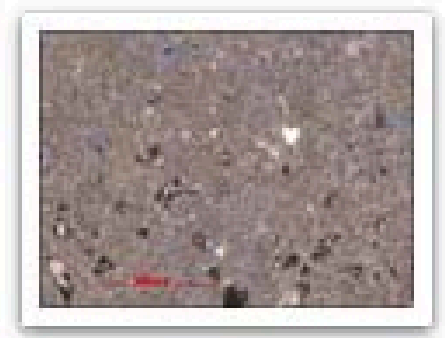

26_WMIKKEL-01 Core 4 11,367' Spi...gs 10X.jpg

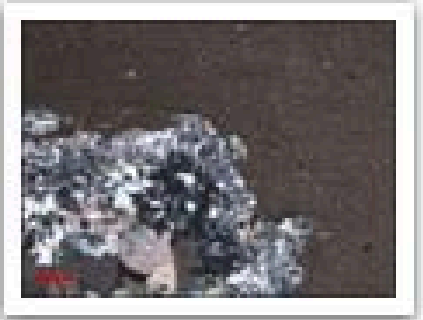

31_WMIKKEL-01 Core 4 11,377' Do...Nicols).jpg

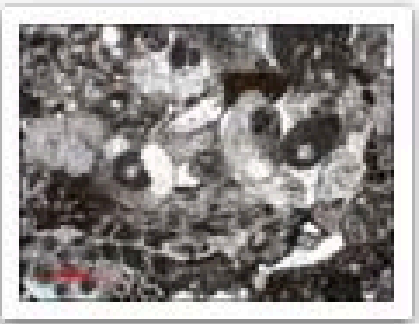

36-WMIKKEL-01 Core 4 $11,385^{\prime}$ Bry...id G 5X.jpg

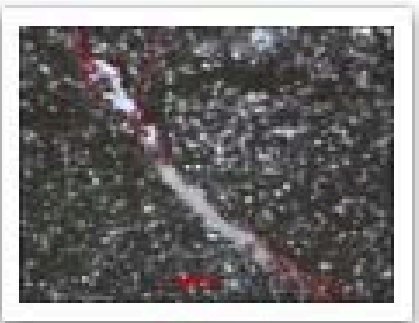

41_WMIKKEL-01 Core 4 11,391 ' VF...lo W 5X.jpg

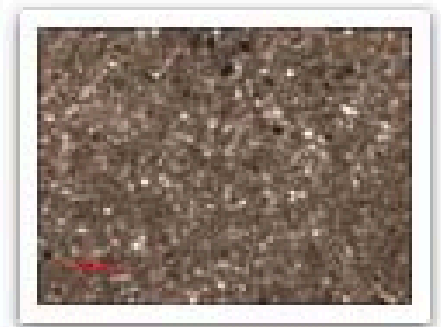

46_WMIKKEL-01 Core 4 11,401 ' Sil...GGPał Repert.38g

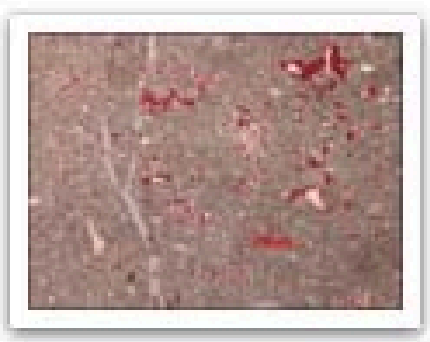

27_WMIKKEL-01 Core 4 $11,370^{\prime}$ Sp...ugs $5 X . j p g$

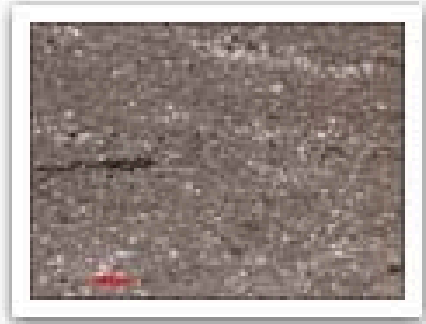

32_WMIKKEL-01 Core 4 11,380' Silt...M 5X .jpg

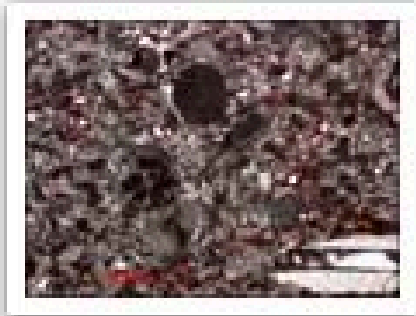

37_WMIKKEL-01 Core 4 $11,385^{\prime}$ Bry...ed) 5X.jpg

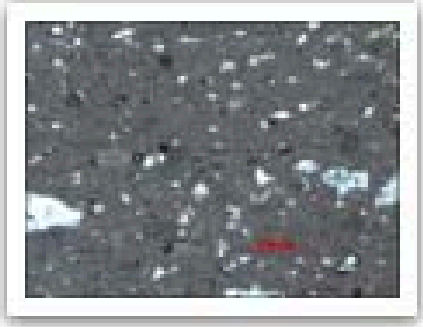

42_WMIKKEL-01 Core 4 $11,394^{\prime}$ Fin...ugs 5X.jpg

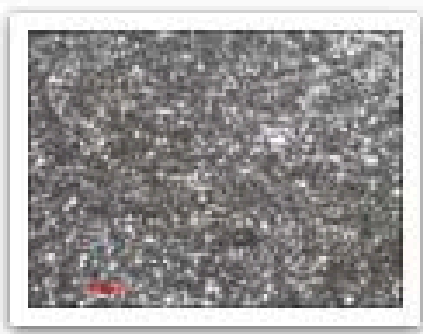

47_WMIKKEL-01 Core 4 $11,403^{\prime}$ Sp...MRP 5X.jpg
28_WMIKKEL-01 Core 4 11,372 Dol...MRP 5X.jpg

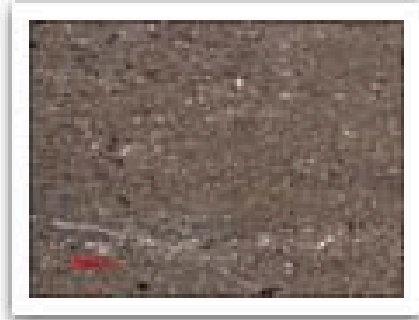

33_WMIKKEL-01 Core 4 11,382' Silt...o M 5X.jpg

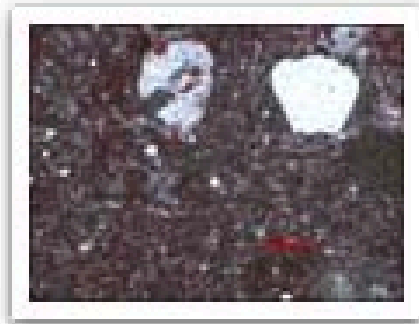

38_WMIKKEL-01 Core 4 11,386' Pel...ed) 5X.jpg

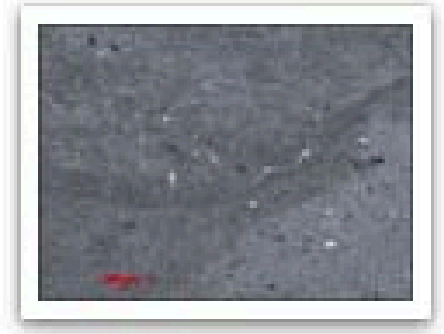

43_WMIKKEL-01 Core 4 $11,397^{\prime}$ Bu...lo M 5X.jpg

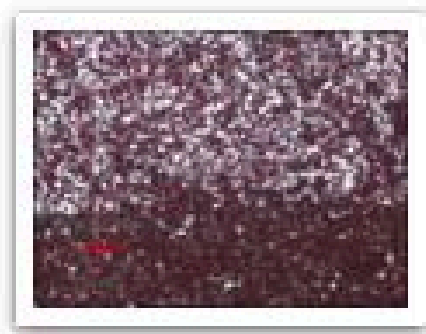

48_WMIKKEL-01 Core 4 $11,403^{\prime}$ V....P-W 5X.jpg
29_WMIKKEL-01 Core 4 $11,373^{\prime}$ Do...Nicols).jpg

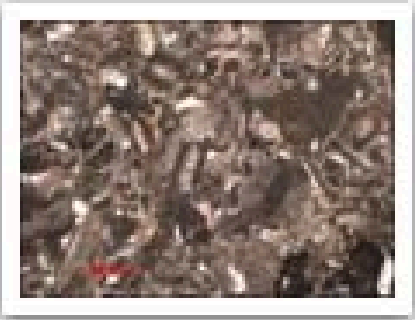

34_WMIKKEL-01 Core 4 11,384' Bry...) G 5X.jpg

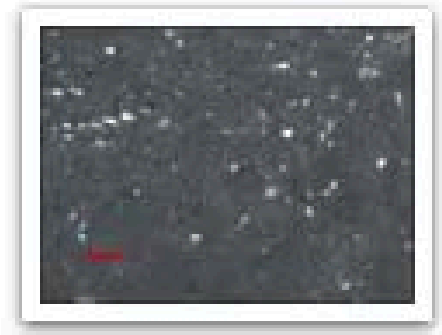

39_WMIKKEL-01 Core 4 11,386' Silt...o M 5X.jpg

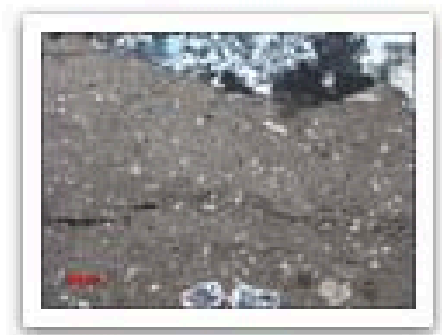

44_WMIKKEL-01 Core 4 $11,400^{\prime}$ Do...Nicols).jpg

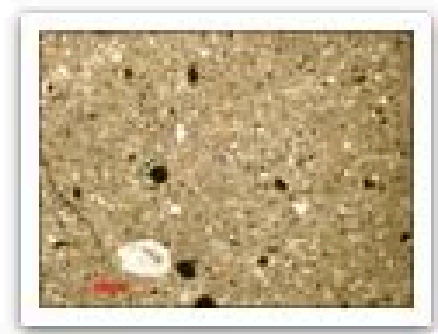

49_WMIKKEL-01 Core 4 $11,405^{\prime}$ Silt...light) .jpg
30_WMIKKEL-01 Core 4 11,373' Do...Nicols).jpg

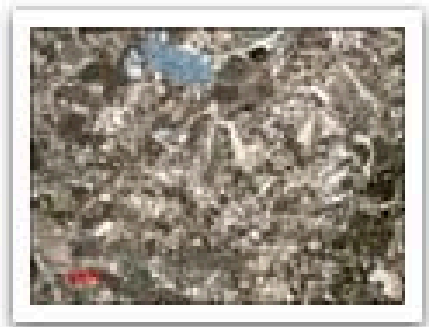

35_WMIKKEL-01 Core 4 $11,384^{\prime}(2) \ldots$-Nicols).jpg

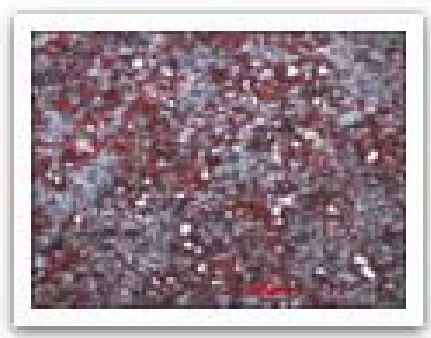

40_WMIKKEL-01 Core 4 $11,389^{\prime} \mathrm{Br}$...tain) 5X.jpg

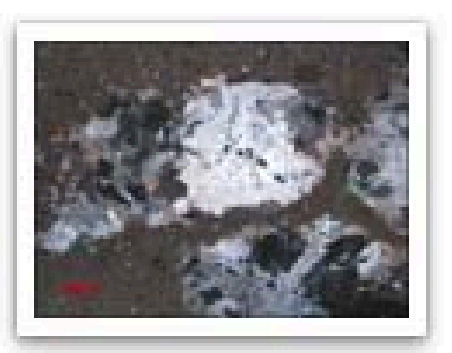

45_WMIKKEL-01 Core 4 $11,400^{\prime}$ Do....Nicols).jpg

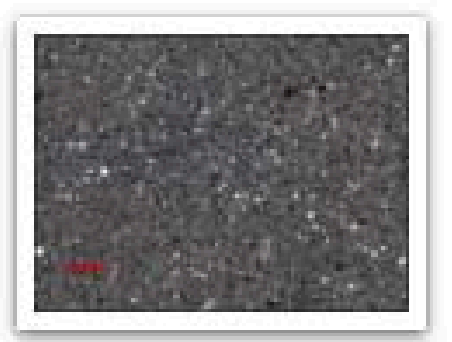

50_WMIKKEL-01 Core 4 $11,405^{\prime}$ Silt2...Pficols).jpg 


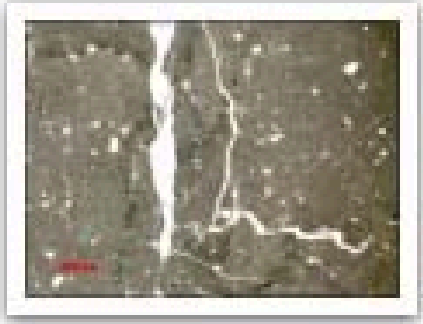

51_WMIKKEL-01 Core 5 $11,585^{\prime}$ Do...racs 5X.jpg

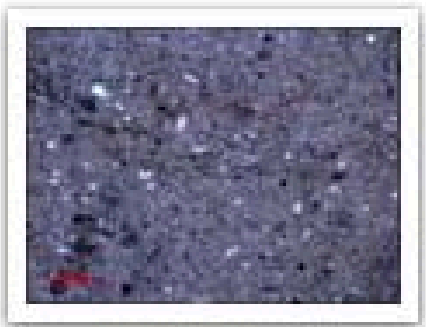

56_WMIKKEL-01 Core 5 11,595' Do...oids 5X.jpg

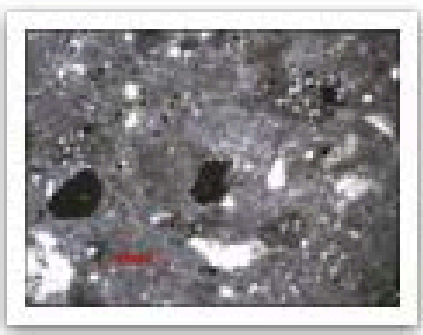

61_WMIKKEL-01 Core 5 11,599' Do...pics 5X.jpg

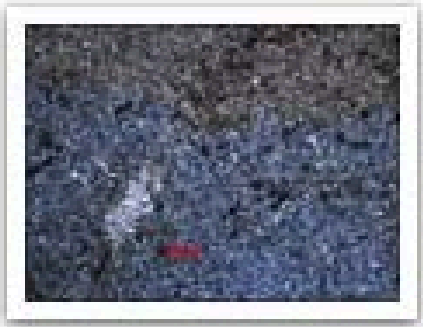

66 WMIKKEL-01 Core 5 $11,615^{\prime}$ Ch...Nicols).jpg

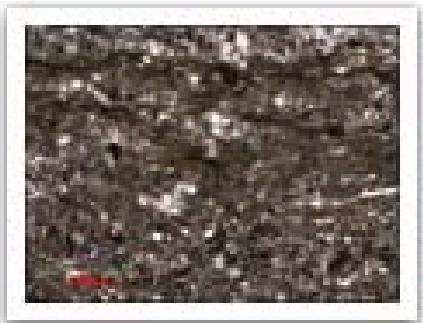

71_WMIKKEL-01 Core 5 (base) 11,6...PataRepgrt.j84

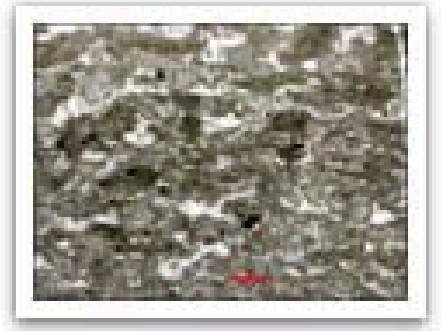

52_WMIKKEL-01 Core 5 11,588' Do...al) 2.5X.jpg

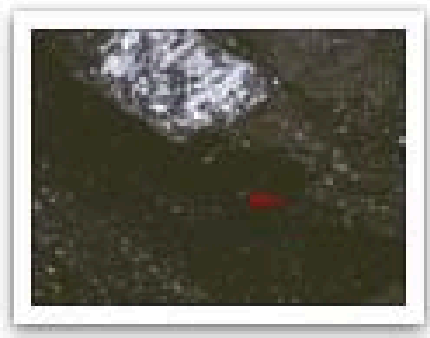

57_WMIKKEL-01 Core 5 11,597' Ch...Photo3).jpg

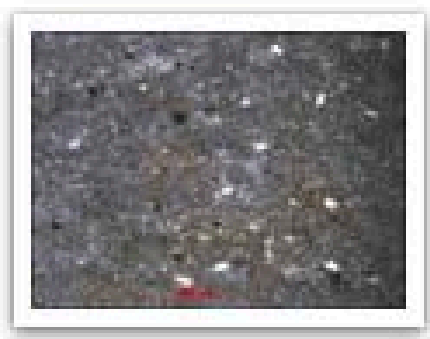

62_WMIKKEL-01 Core 5 $11,602^{\prime}$ Do...In) 10X.jpg

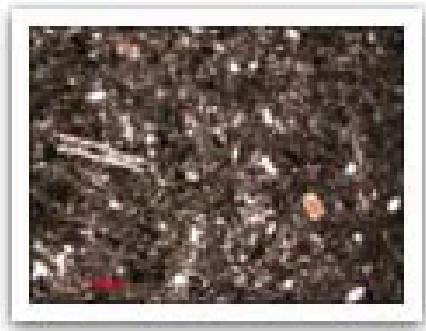

67_WMIKKEL-01 Core 5 $11,617^{\prime}$ Sp...lo W 5X.jpg

72_WMIKKEL-01 Core 6 (top) $11,68 \ldots \mathrm{M} \mathrm{2.5X.jpg}$
68_WMIKKEL-01 Core 5 11,620 ' Silt...ams 5X.jpg

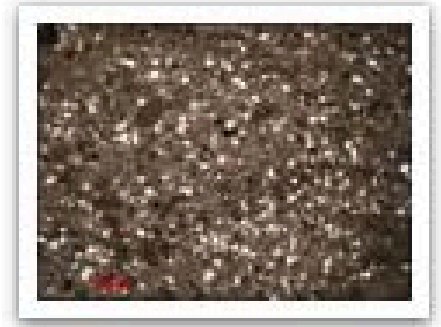

53_WMIKKEL-01 Core 5 $11,590^{\prime}$ Do...ins. $5 X . j p g$

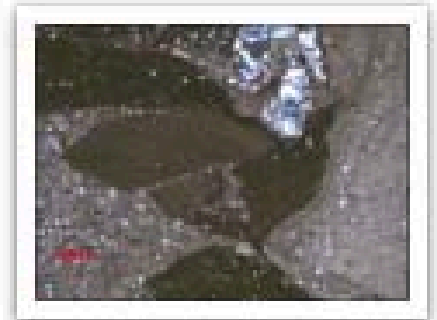

58_WMIKKEL-01 Core 5 11,597' Co...Is) 2.5X.jpg

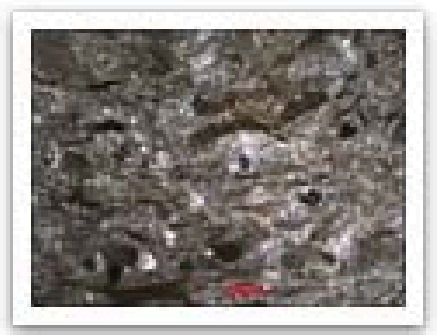

63_WMIKKEL-01 Core 5 $11,605^{\prime}$ Do...ps 2.5X.jpg
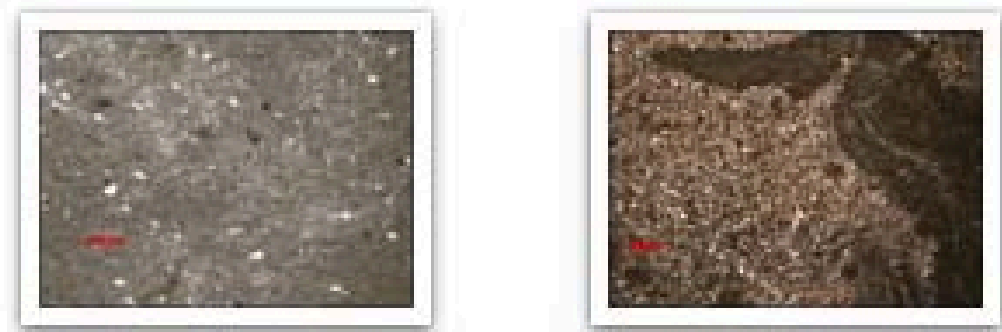

69_WMIKKEL-01 Core 5 $11,625^{\prime}$ Silt...n 2.5X.jpg

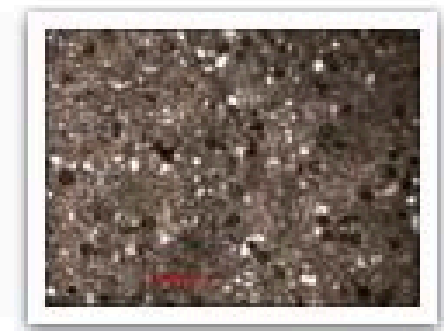

73_WMIKKEL-01 Core 6 $11,684^{\prime}$ V..... M 2.5X.jpg

54_WMIKKEL-01 Core 5 11,591' Do...In) 10X.jpg

59_WMIKKEL-01 Core 5 11,597' Co...Photo4).jpg

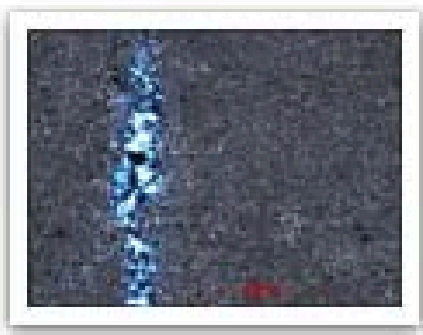

64_WMIKKEL-1 Core 5 $11,608^{\prime}$ Do....Nicols).jpg

74_WMIKKEL-01 Core 6 $11,687^{\prime}$ V....ubbles).jpg
65 WMIKKEL-01 Core 5 $11,612^{\prime}$ Do...s. 2.5X.jpg

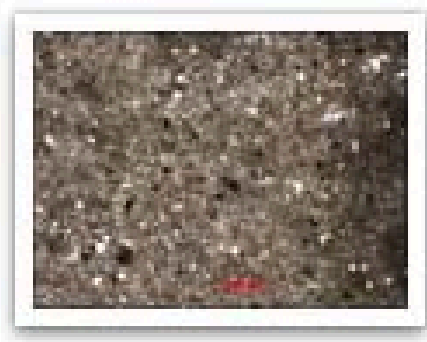

70_WMIKKEL-01 Core 5 11,627' Silt...o M 5X.jpg

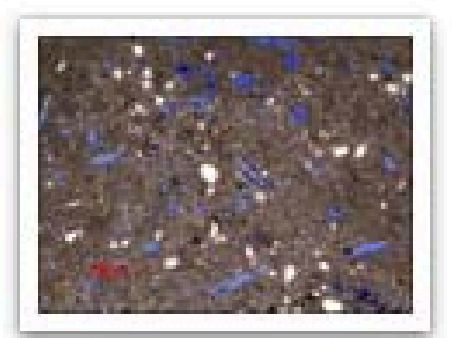

75_WMIKKEL-01 Core 6 $11,690^{\prime}$ Silt.... $0^{25} \mathrm{~W}$ 5X.jpg 


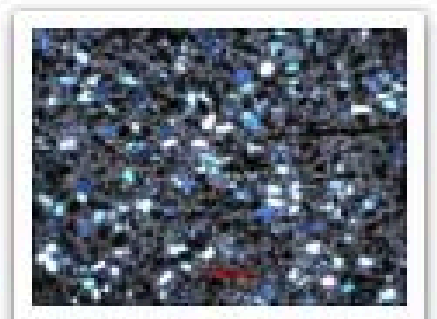

76_WMIKKEL-01 Core 6 $11,693^{\prime}$ V....-Nicols).jpg

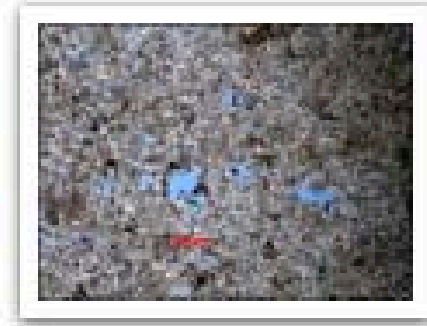

81_WMIKKEL-01 Core 6 $11,707^{\prime}$ Do...ugs) 5X.jpg

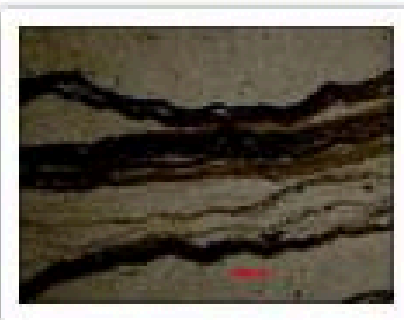

86 WMIKKEL-01 Core 6 $11,719^{\prime}$ Sp...ins. 5X.jpg

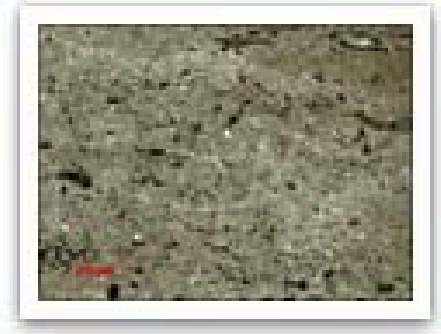

91_WMIKKEL-01 Core 6 $11,729^{\prime}$ Sp...lo W 5X.jpg

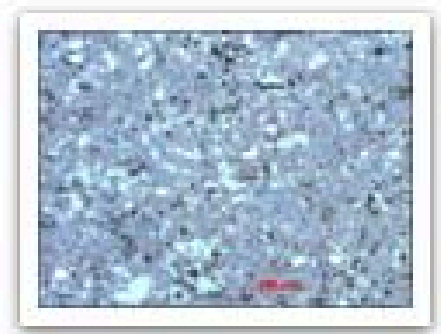

96_WMIKKEL-01 Core 4 (Set 2) $11, \ldots M$ GD W Therort 384

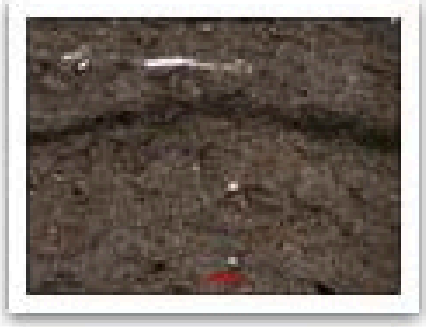

77_WMIKKEL-01 Core 6 $11,697^{\prime}$ Do...ics) 5X.jpg

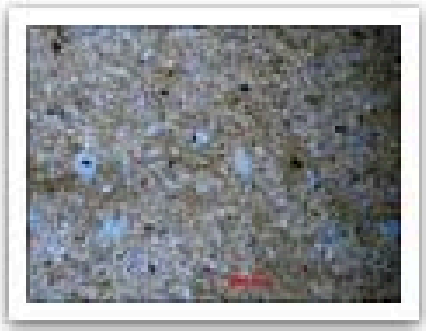

82_WMIKKEL-01 Core 6 $11,708^{\prime}$ Spi...gy) 5X.jpg

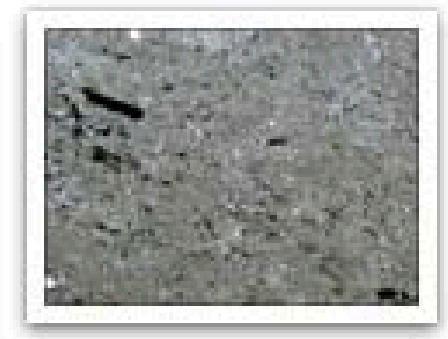

87_WMIKKEL-01 Core 6 $11,722^{\prime}$ Ex...ritic) 5X.jpg

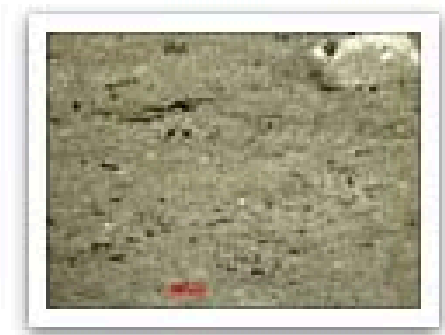

92_WMIKKEL-01 Core 6 $11,729^{\prime}(2)$..W 2.5X.jpg

97_WMIKKEL-01 Core 4 (Set 2) $11, \ldots$ lo W 5X.jpg
93_WMIKKEL-01 Core 2 (Set 2) $11, \ldots$ ed) $2.5 X . j p g$

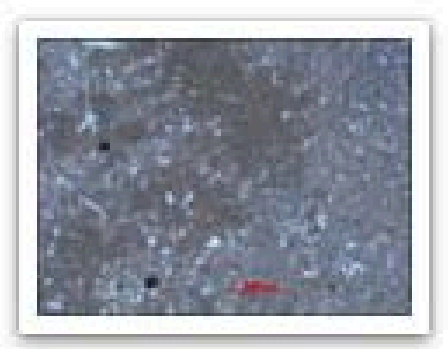

78_WMIKKEL-01 Core 6 11,699' Spi...gy) 5X.jpg

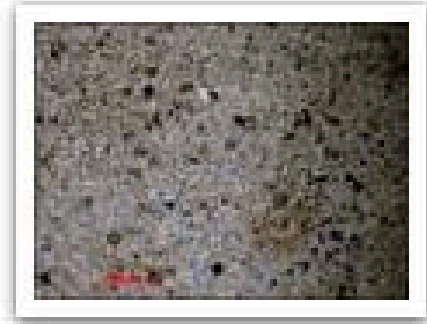

83_WMIKKEL-01 Core 6 $11,710^{\prime}$ Do...ugs) 5X.jpg

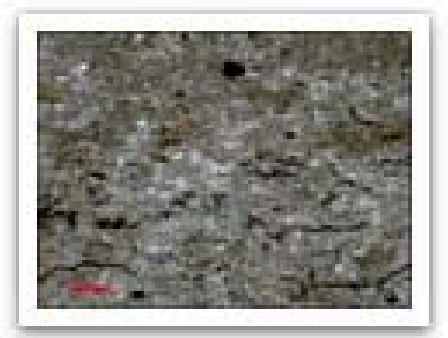

88_WMIKKEL-01 Core 6 $11,725^{\prime}$ Do...ins. 5X.jpg
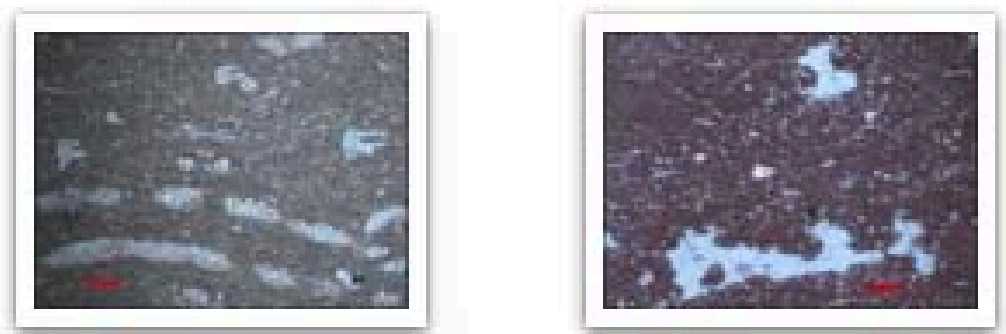

94_WMIKKEL-01 Core 3 (Set 2) 11,...e W 5X.jpg

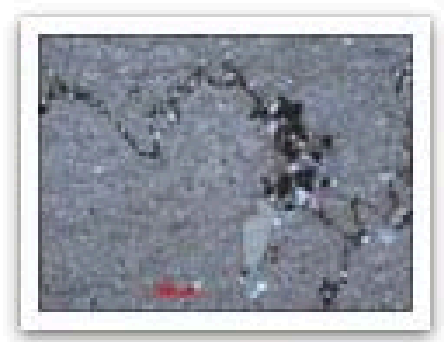

98_WMIKKEL-01 Core 4 (Set 2) $11, \ldots . f \times \ln ) 5 X . j p g$

79_WMIKKEL-01 Core 6 11,701' Spi...y) 2.5X.jpg

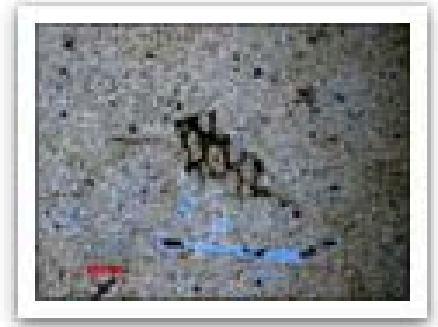

84_WMIKKEL-01 Core 6 $11,715^{\prime}$ Spi...ugs 5X.jpg

89_WMIKKEL-01 Core 6 $11,728^{\prime}$ Do...tained).jpg

99_WMIKKEL-01 Core 4 (Set 2) $11, \ldots$ lites 5X.jpg
MIKKEL-01 Core 6 $11,728^{\prime}$ Do...ned) 5X.jpg

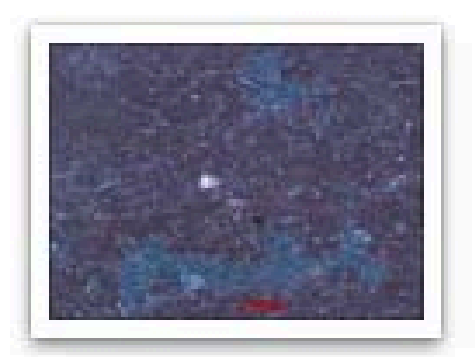

95_WMIKKEL-01 Core 3 (Set 2) 11,..-Nicols).jpg

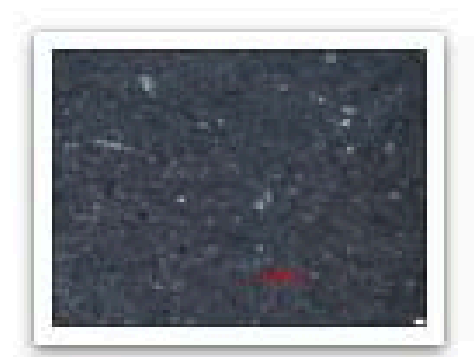

100_WMIKKEL-01 Core 4 (Set 2) 1 ... of 24 cols).jpg 


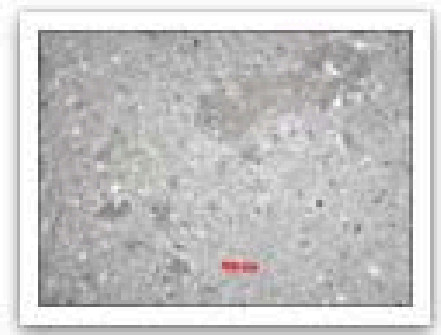

101_WMIKKEL-01 Core 4 (Set 2) 1...-M 2.5X.jpg

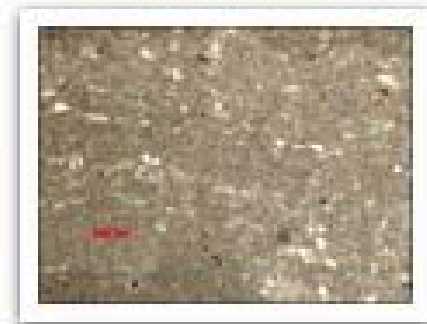

106_WMIKKEL-01 Core 4 (Set 2) 1...Sand 5X.jpg

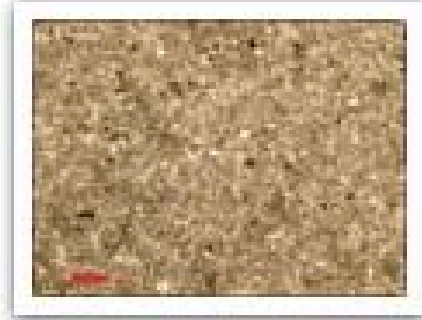

111_WMIKKEL-01 Core 5 (Set 2) 1...Vugs 5X.jpg

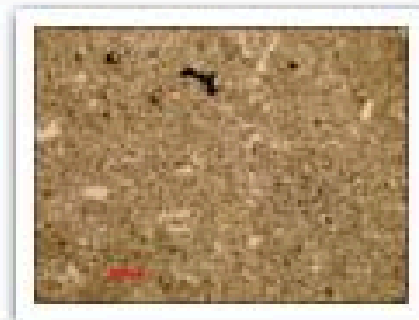

116_WMIKKEL-01 Core 6 (Set 2) 1 GMc patajRegort 384
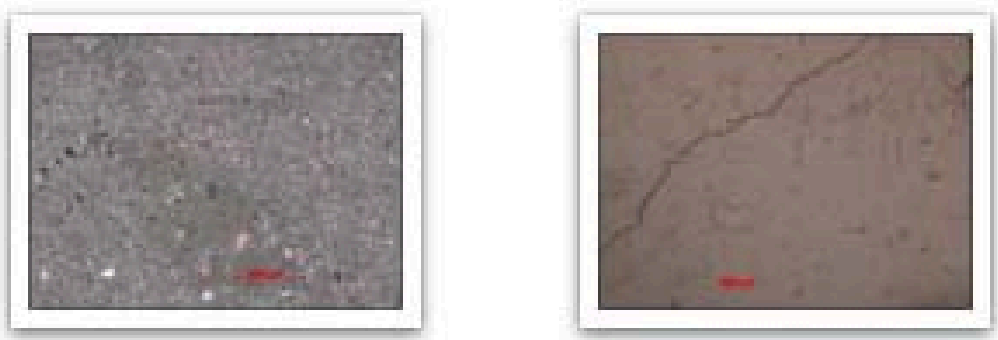

102_WMIKKEL-01 Core 4 (Set 2) 1...lled) 5X.jpg

103_WMIKKEL-01 Core 4 (Set 2) 1...taining).jpg

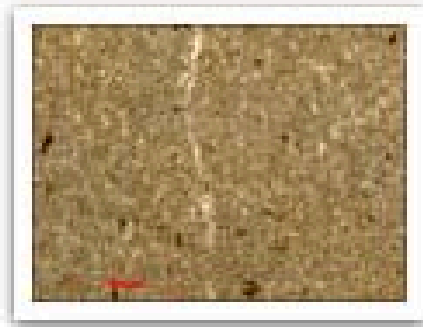

107_WMIKKEL-01 Core 5 (Set 2) 1...-Nicols).jpg

108_WMIKKEL-01 Core 5 (Set 2) 1...rac 10X.jpg

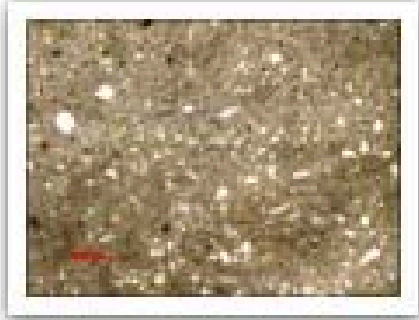

112_WMIKKEL-01 Core 6 (Set 2) 1...ggy) 5X.jpg

113_WMIKKEL-01 Core 6 (Set 2) 1...ggy) 5X.jpg

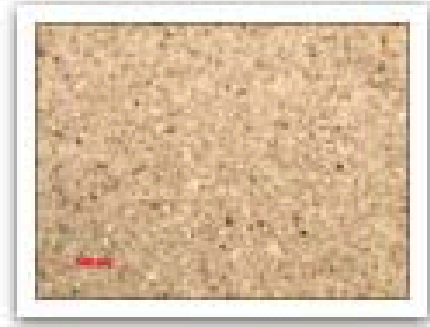

117_WMIKKEL-01 Core 6 (Set 2) 1...lo W 5X.jpg

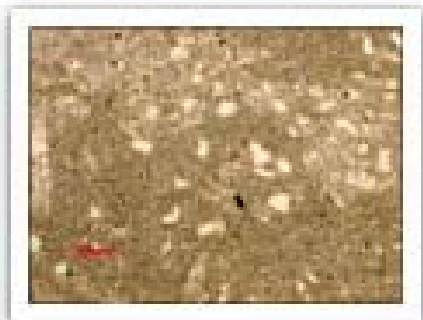

118_WMIKKEL-01 Core 6 (Set 2) 1...too thin).jpg

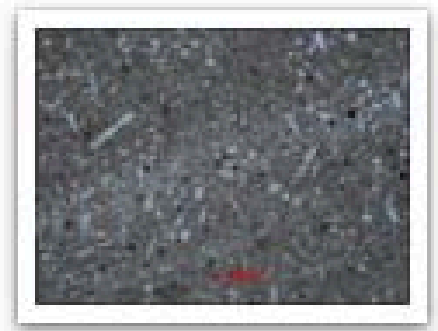

104_WMIKKEL-01 Core 4 (Set 2) 1...Frac 5X.jpg
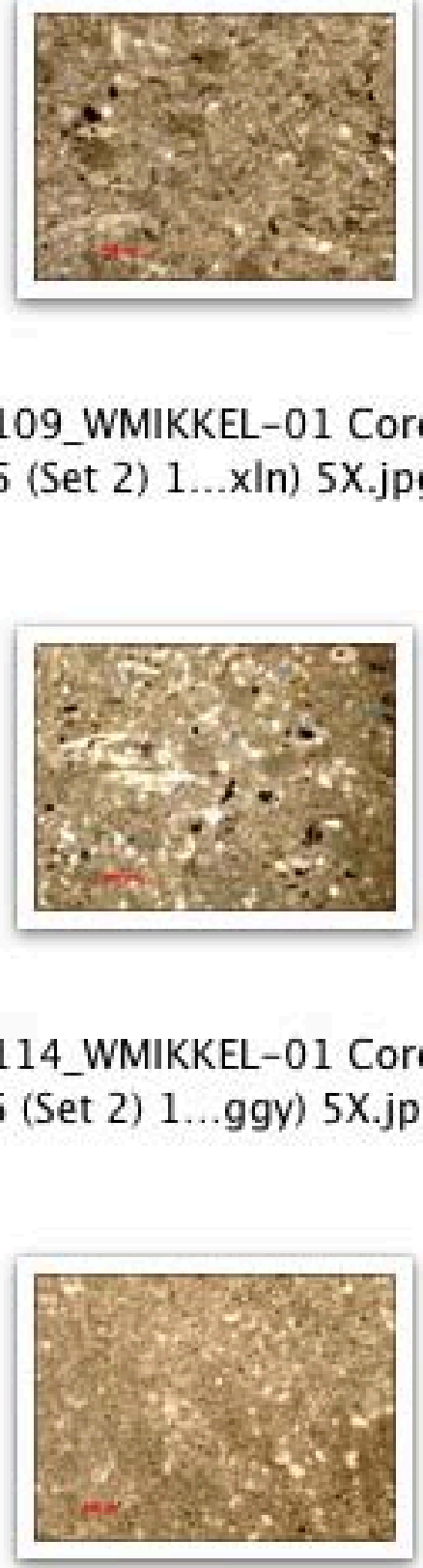

109_WMIKKEL-01 Core 5 (Set 2) 1...x In) 5X.jpg

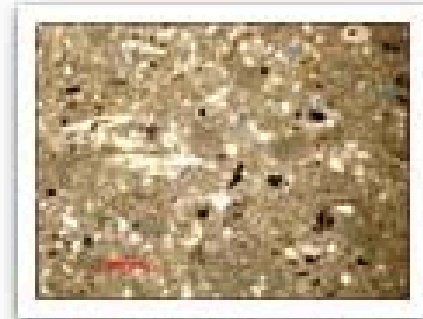

114_WMIKKEL-01 Core 6 (Set 2) 1...ggy) 5X.jpg

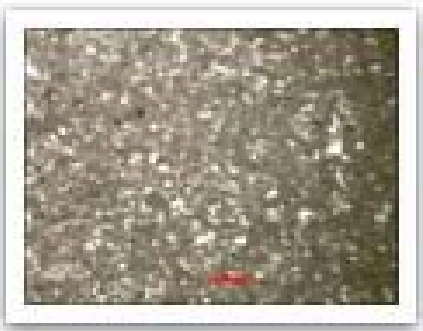

105_WMIKKEL-01 Core 4 (Set 2) 1...Vugs 5X.jpg

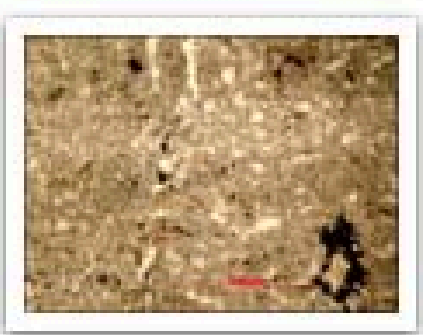

110_WMIKKEL-01 Core 5 (Set 2) 1...rac 10X.jpg

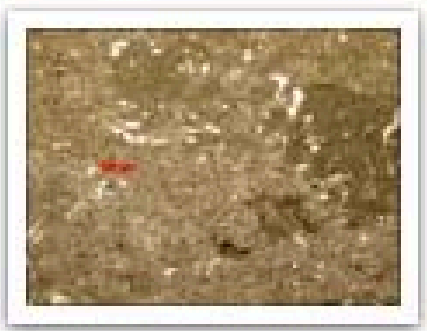

115_WMIKKEL-01 Core 6 (Set 2) 1...gy) 2.5X.jpg

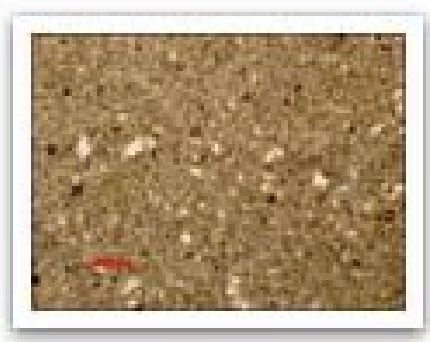

119_WMIKKEL-01 Core 6 (Set 2) 1...oor T.S.).jpg
120_WMIKKEL-01 Core 6 (Set 2) 1...po 25 W 5X.jpg 